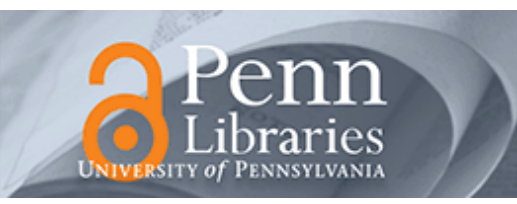

University of Pennsylvania

ScholarlyCommons

$9-18-2008$

\title{
Effective-medium theory for infinite-contrast two-dimensionally periodic linear composites with strongly anisotropic matrix behavior: Dilute limit and crossover behavior
}

François Willot

Commissariat à l'Énergie Atomique, Bruyères-le-Châtel

Yves-Patrick Pellegrini

Commissariat à l'Énergie Atomique, Bruyères-le-Châtel

Martín I. Idiart

Département de Mécanique, École Polytechnique; University of Pennsylvania

Pedro Ponte Castañeda

Département de Mécanique, École Polytechnique; University of Pennsylvania

Follow this and additional works at: https://repository.upenn.edu/meam_papers

Part of the Mechanical Engineering Commons

\section{Recommended Citation}

Willot, François; Pellegrini, Yves-Patrick; Idiart, Martín I.; and Castañeda, Pedro Ponte, "Effective-medium theory for infinite-contrast two-dimensionally periodic linear composites with strongly anisotropic matrix behavior: Dilute limit and crossover behavior" (2008). Departmental Papers (MEAM). 263.

https://repository.upenn.edu/meam_papers/263

Suggested Citation:

F. Willot, Y. Pellegrini, M.I. Idiart and P.P. Castañeda. (2008). "Effective-medium theory for infinite-contrast twodimensionally periodic linear composites with strongly anisotropic matrix behavior: Dilute limit and crossover behavior." Physical Review B. 78, 104111.

(C) 2008 The American Physical Society

http://dx.doi.org/10.1103/PhysRevB.78.10411

This paper is posted at ScholarlyCommons. https://repository.upenn.edu/meam_papers/263

For more information, please contact repository@pobox.upenn.edu. 


\title{
Effective-medium theory for infinite-contrast two-dimensionally periodic linear composites with strongly anisotropic matrix behavior: Dilute limit and crossover behavior
}

\author{
Abstract \\ The overall behavior of a two-dimensional lattice of voids embedded in an anisotropic elastic matrix is \\ investigated in the limit of vanishing porosity $f$. An effective-medium model (of the Clausius-Mossoti \\ type), which accounts for elastic interactions between neighboring voids, is compared to fast Fourier \\ transform numerical solutions and, in the limits of infinite anisotropy, to exact results. A crossover \\ between regular and singular dilute regimes is found, driven by a characteristic length which depends on $f$ \\ and on the anisotropy strength. The singular regime, where the leading dilute correction to the elastic \\ moduli is an $\mathrm{O}\left(f^{1 / 2}\right)$, is related to strain localization and to change in character-from elliptic to \\ hyperbolic-of the governing equations. \\ Disciplines \\ Engineering | Mechanical Engineering

\section{Comments} \\ Suggested Citation: \\ F. Willot, Y. Pellegrini, M.I. Idiart and P.P. Castañeda. (2008). "Effective-medium theory for infinite-contrast \\ two-dimensionally periodic linear composites with strongly anisotropic matrix behavior: Dilute limit and \\ crossover behavior." Physical Review B. 78, 104111. \\ (C) 2008 The American Physical Society \\ http://dx.doi.org/10.1103/PhysRevB.78.10411
}




\title{
Effective-medium theory for infinite-contrast two-dimensionally periodic linear composites with strongly anisotropic matrix behavior: Dilute limit and crossover behavior
}

\author{
François Willot, ${ }^{1, *}$ Yves-Patrick Pellegrini, ${ }^{1, \dagger}$ Martín I. Idiart, ${ }^{2,3, \ddagger}$ and Pedro Ponte Castañeda ${ }^{2,3,8}$ \\ ${ }^{1}$ Département de Physique Théorique et Appliquée, Commissariat à l'Énergie Atomique, Bruyères-le-Châtel, F-91297 Arpajon, France \\ ${ }^{2}$ Laboratoire de Mécanique des Solides, CNRS UMR 7649, Département de Mécanique, École Polytechnique, 91128 Palaiseau \\ Cedex, France \\ ${ }^{3}$ Department of Mechanical Engineering and Applied Mechanics, School of Engineering and Applied Science, University of Pennsylvania, \\ Philadelphia, Pennsylvania 19104-6315, USA
}

(Received 16 April 2008; revised manuscript received 24 July 2008; published 18 September 2008)

\begin{abstract}
The overall behavior of a two-dimensional lattice of voids embedded in an anisotropic elastic matrix is investigated in the limit of vanishing porosity $f$. An effective-medium model (of the Clausius-Mossoti type), which accounts for elastic interactions between neighboring voids, is compared to fast Fourier transform numerical solutions and, in the limits of infinite anisotropy, to exact results. A crossover between regular and singular dilute regimes is found, driven by a characteristic length which depends on $f$ and on the anisotropy strength. The singular regime, where the leading dilute correction to the elastic moduli is an $O\left(f^{1 / 2}\right)$, is related to strain localization and to change in character-from elliptic to hyperbolic — of the governing equations.
\end{abstract}

DOI: 10.1103/PhysRevB.78.104111

PACS number(s): 46.05.+b, 46.15.Ff

\section{INTRODUCTION}

Effective-medium approximations (EMAs) for nonlinear composites $^{1-10}$ (i.e., multiphase materials), which aim to predict their overall (i.e., macroscopic) behavior, are pushed to their limits of validity as the nonlinearity and/or the heterogeneity contrast become large. ${ }^{7}$ Typical examples of this sort of phenomenon in continuum mechanics include porous, ${ }^{11-13}$ and rigidly reinforced, ${ }^{13,14}$ plastic or nonlinearly elastic media. In the idealized model of perfect plasticity, plastic material flow takes place at constant stress intensity (the yield stress). In such circumstances, the flow preferentially concentrates (localizes) in shear bands. ${ }^{15}$ Formally, these shear bands are closely related to other types of minimal breakdown manifolds in heterogeneous media (mechanical systems, as well as nonlinear electrical networks). ${ }^{16-18}$ However, nonlinear EMAs which address plasticity rely on a quasiequilibrium hypothesis, which means that the characteristic time of an individual "breakdown" (or slip) event is longer than that of wave propagation through the medium ${ }^{19}$ (in nonlinear dielectrics, such conditions are met, as well as in the reversible diode network experiment ${ }^{20}$ ). Plastic deformation being a strongly irreversible process, applications of such EMAs to plasticity should therefore be restricted to incipient deformation in an incremental framework. ${ }^{12,15}$ Nonetheless, upon neglecting elasticity and assuming plastic incompressibility, the quasiequilibrium hypothesis may extend to full-grown deformations, such as in the slip-line theory of perfect plasticity, but the governing equations are then hyperbolic. $^{15}$

Efficient nonlinear EMAs rely on the use of an underlying linear comparison medium, ${ }^{21,22}$ which may consist in a "secant" (isotropic) approximation ${ }^{23}$ to the nonlinear response of the composite. In the most recent approaches the comparison medium is anisotropic, of direction determined by the applied field, ${ }^{6}$ and of strength being consistently determined by the covariance tensors of the local fields in the phases. ${ }^{8-10}$ How these methods cope with localization at the overall level in heterogeneous media is not well understood (see Ref. 24 and references therein).

To address this issue, this paper is devoted to the signature of incipient localization in an EMA for periodic composites. $^{25}$ Because for periodic media efficient methods of solution have been developed, ${ }^{24,26-34}$ our focus here is on such materials. Thus, a system consisting of a twodimensional (2D) periodic lattice of voids embedded in a deformable matrix is considered. Aimed at understanding the hallmarks of localization in the underlying linear medium of nonlinear EMAs, we focus on the case of an elastically anisotropic linear matrix of variable anisotropy. ${ }^{24,35}$

The problem, described in Sec. II, admits an exact analytical solution in the particular case of infinite anisotropy, where the governing equations acquire an hyperbolic character. ${ }^{24,35}$ As a consequence, the overall elastic moduli depend on the porosity $f$ (the volume fraction of voids) as powers of $f^{1 / 2}$, in particular in the dilute limit $f \rightarrow 0 .^{24}$ This result is at odds with usual effective-medium results, in which the first correction to a homogeneous medium is an $O(f),,^{30,36}$ due to its proportionality to the number of inclusions. $^{37}$

But for finite anisotropy, the governing equations are elliptic, and no exact solution is available. The crossover to the regime of high anisotropy and its link to localization, of direct interest for nonlinear EMAs, and more generally for understanding the nature of the macroscopic yield transition, ${ }^{17}$ are investigated hereafter. For lack of exact solutions, the following are compared: (i) quasiexact numerical results obtained by fast Fourier transform (FFT) calculations, (ii) outcomes of an EMA for linear periodic media whose predictive capabilities are assessed, and (iii) the exact results of Ref. 24. The case of a nonlinear (visco-)plastic matrix is examined elsewhere. ${ }^{38}$

The notation used is as follows: A denotes a tensor of components $A_{i j k l}$, the sans-serif $\mathbf{a}$ is the tensor of components $a_{i j}$ (except for the strain and stress $\boldsymbol{\varepsilon}$ and $\boldsymbol{\sigma}$, and the strain polarization $\boldsymbol{\tau}$, written in boldface), and the boldface $\mathbf{a}$ is the 


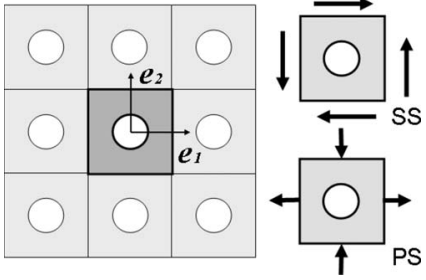

FIG. 1. Left, periodic porous medium with unit cell and reference axes. Right, black arrows depict eigenmodes of strain: simple shear (SS) and pure shear (PS).

vector of components $a_{i}$. A colon denotes a double contraction, e.g., $\mathrm{A}: \mathbb{B}$ has components $A_{i j m n} B_{m n k l}$, etc. For convenience, indices $i=x, y$ or 1,2 are used indifferently hereafter.

\section{PROBLEM FORMULATION}

The composite, described in Fig. 1, consists of an elastic matrix (phase $\alpha=1$, of volume fraction $c^{(1)}=1-f$ ), containing a square array of voided cylinders of radius $a$ (phase $\alpha$ $=2$, of volume fraction $c^{(2)} \equiv f=\pi a^{2}$ ). Here and henceforth, the size of the unit cell is $L=1$. A set of duality relations ${ }^{39}$ allows one to translate the following results for the overall behavior of this porous medium in the context of rigidly reinforced composites, which is another interesting case of infinite elastic contrast.

In the composite $\boldsymbol{\sigma}(\mathbf{x})=\mathbb{L}_{(}(\mathbf{x}): \boldsymbol{\varepsilon}(\mathbf{x})$, where $\mathbb{L}_{(}(\mathbf{x})$ is the position-dependent elasticity tensor, of components $L_{i j k l}$ $=L_{i j l k}=L_{k l i j}$. The equilibrium equation $\partial_{i} \sigma_{i j}=0$ holds, and the strain derives from the displacement $\mathbf{u}$ as $\varepsilon_{i j}=\left(\partial_{i} u_{j}+\partial_{j} u_{i}\right) / 2$ (small perturbations are assumed). In two dimensions, $\varepsilon_{x x}$ $=\partial_{x} u_{x}, \varepsilon_{y y}=\partial_{y} u_{y}$, and $\varepsilon_{x y}=\left(\partial_{x} u_{y}+\partial_{y} u_{x}\right) / 2$. In the voids, $\mathrm{L}(x)$ $=\mathbb{L}^{(2)} \equiv 0$, the stress vanishes and the strain is arbitrary: any continuation matching the displacements at the void boundaries is admissible. Only its volume average over the void is physically relevant.

The matrix material can be thought of as a "mixture" of two basic types of anisotropic media: ${ }^{24}$ (i) one where the eigendirections of anisotropy coincide with the reference axes of unit vectors $e_{1} \equiv e^{x}$ and $e_{2} \equiv e^{y}$, and (ii) one where they coincide with the diagonals (see Fig. 1). Such a medium is invariant under the dihedral point-symmetry group $D_{4}$. Then, its elastic tensor $\mathbb{L}^{(1)}$ is of the form

$$
\left(L_{1111}+L_{1122}\right) \mathrm{J}+2 L_{1212} \mathbb{E}^{\mathrm{SS}}+\left(L_{1111}-L_{1122}\right) \mathbb{E}^{\mathrm{PS}},
$$

where $J, \mathbb{E}^{\mathrm{PS}, \mathrm{SS}}$ are mutually orthogonal projectors defined by (I, of components $\delta_{i j}$, is the $2 \times 2$ identity matrix)

$$
\begin{gathered}
\mathbb{J} \equiv(1 / 2) \mathbf{I} \otimes \mathbf{I}, \\
\mathbb{E}^{\mathrm{SS}, \mathrm{PS}} \equiv(1 / 2) \mathbf{e}^{\mathrm{SS}, \mathrm{PS}} \otimes \mathbf{e}^{\mathrm{SS}, \mathrm{PS}} .
\end{gathered}
$$

The identity is $I=J+\mathbb{E}_{4}^{S S}+\mathbb{E}_{A}^{P S}$. The definitions involve the so-called simple shear (SS) and pure shear (PS) eigenmodes of deformation:

$$
\mathbf{e}^{\mathrm{SS}} \equiv \mathbf{e}_{1} \otimes \mathbf{e}_{2}+\mathbf{e}_{2} \otimes \mathbf{e}_{1}
$$

$$
\mathbf{e}^{\mathrm{PS}} \equiv \mathbf{e}_{1} \otimes \mathbf{e}_{1}-\mathbf{e}_{2} \otimes \mathbf{e}_{2},
$$

such that $\mathbb{E}^{S S, P S}: \mathbf{e}^{\mathrm{SS}, \mathrm{PS}}=\mathbf{e}^{\mathrm{SS}, \mathrm{PS}}$. Their eigenvectors are related by a $45^{\circ}$ rotation (see Fig. 1 ). Also $J: \mathbf{I}=\mathbf{I}$. This decomposition relates to that of a $2 \times 2$ symmetric tensor a into one equibiaxial (i.e., compressive) mode and two orthogonal shear modes:

$$
\mathbf{a}=a_{m} \mathbf{I}+a_{\mathrm{SS}} \mathbf{e}^{\mathrm{SS}}+a_{\mathrm{PS}} \mathbf{e}^{\mathrm{PS}},
$$

of respective components $a_{m} \equiv\left(a_{x x}+a_{y y}\right) / 2$ (called mean stress or strain hereafter), and $a_{\mathrm{SS}} \equiv a_{x y}, a_{\mathrm{PS}} \equiv\left(a_{x x}-a_{y y}\right) / 2$. Thus, in the matrix we write

$$
\mathrm{L}_{(}(x)=\mathbb{L}_{4}^{(1)} \equiv 2 \kappa \mathbb{J}+2 \lambda \mathbb{E}^{\mathrm{SS}}+2 \mu \mathbb{E}^{\mathrm{PS}} .
$$

$\kappa$ is the bulk compressibility modulus, and $\lambda, \mu$ are in-plane anisotropic shear moduli. With this medium of a special orthotropic type, the interpretation of the $2 \mathrm{D}$ problem as a limiting one of plane stress $\left(\sigma_{x z}=\sigma_{y z}=\sigma_{z z}=0, \varepsilon_{z z} \neq 0\right)$ or of plane strain $\left(\varepsilon_{x z}=\varepsilon_{y z}=\varepsilon_{z z}=0, \sigma_{z z} \neq 0\right)$ is irrelevant from a theoretical standpoint, although the expression in terms of $\kappa$ and of $\mu$ of the Young modulus and Poisson ratio relative to the pure shear mode differ in both cases. ${ }^{40}$

With applications to volume-preserving plastic deformation in mind, this study mostly focuses on the limiting case of an incompressible matrix for which $\kappa=\infty$. Introducing in this limit the dimensionless anisotropy ratio $k=\lambda / \mu$, the medium is isotropic when $k=1$, and is infinitely anisotropic when either $k=0$ or $k=\infty$. In each of the latter limits, the medium possesses one infinitely hard and one infinitely soft eigenmodes of strain: when $k=0$ (i.e., $\lambda=0$ or $\mu=\infty$ ) the medium is soft for SS loadings and resists PS loadings, whereas when $k=\infty$ (i.e., $\lambda=\infty$ or $\mu=0$ ) the medium is soft for PS loadings and resists SS loadings. We accordingly call these loading modes "hard" and "soft" hereafter. This model provides a convenient framework for studying the coupling between porosity and localization.

Volume averages over the whole system are denoted by brackets $\langle\cdot\rangle$. Hereafter, $\langle\cdot\rangle^{(\alpha)}$ stands for a volume average over phase $\alpha$. By definition, the effective (overall) tensor of elastic moduli, $\widetilde{\mathbb{L}}$, relates the macroscopic strain $\overline{\boldsymbol{\varepsilon}}=\langle\boldsymbol{\varepsilon}\rangle$ to the macroscopic stress $\overline{\boldsymbol{\sigma}}=\langle\boldsymbol{\sigma}\rangle$ :

$$
\overline{\boldsymbol{\sigma}}=\tilde{\mathrm{L}}: \overline{\boldsymbol{\varepsilon}} .
$$

The square void lattice also being invariant under $D_{4}$ and $\tilde{\mathbb{L}}_{4}$ is of a form analogous to Eq. (5), where $\kappa, \lambda$, and $\mu$ are replaced by the effective moduli $\widetilde{\kappa}, \tilde{\lambda}$, and $\tilde{\mu}$. The latter are the main quantities of interest. They depend on $\kappa, \lambda, \mu$, and $f$. Even when $\kappa=\infty$, the effective modulus $\widetilde{\kappa}$ is finite for the porous medium. Then, the normalized moduli $\tilde{\lambda} / \lambda, \tilde{\mu} / \mu \mathrm{de}-$ pend only on $k$, and on $f$. Convenient normalizations for $\tilde{\kappa}$ are $\tilde{\kappa} / \mu$ when $\lambda \rightarrow \infty$, or $\tilde{\kappa} / \lambda$ when $\mu \rightarrow \infty$.

\section{FULL-FIELD FFT APPROACH}

\section{A. Numerical method}

Full-field numerical solutions of the problem are obtained using the Fourier transform method, ${ }^{41}$ applied to linear com- 
posites. The method amounts to solving iteratively the Lippmann-Schwinger equation for the strain, ${ }^{42}$

$$
\begin{gathered}
\boldsymbol{\varepsilon}(\mathbf{x}) \equiv \overline{\boldsymbol{\varepsilon}}+\int \mathrm{d}^{2} y \mathrm{G}(\mathbf{x}-\mathbf{y}): \boldsymbol{\tau}(\mathbf{y}), \\
\boldsymbol{\tau}(\mathbf{x}) \equiv\left[\mathbb{L}(\mathbf{x})-\mathbb{L}^{(0)}\right]: \boldsymbol{\varepsilon}(\mathbf{x}),
\end{gathered}
$$

where $\mathbb{L}^{(0)}$ is some arbitrary background elastic tensor. The position-dependent elastic tensor of the medium, $\mathrm{L}(x)$, is 0 $\left(=\mathrm{L}^{(2)}\right)$ in the void and $\mathrm{L}^{(1)}$ in the matrix. In all the numerical calculations of the paper, the latter is assumed nearly incompressible with $\kappa \simeq 10^{3}$, and no appreciable differences were observed for $\kappa \simeq 10^{2}$. The tensor $G$ is the periodic Green function of the background medium, such that $\int d^{2} x \mathrm{G}(x)=0$. In the method, the convolution in Eq. (7a) is evaluated in Fourier space, whereas Eq. (7b) is computed in direct space. The unit cell of the periodic composite is finely discretized as an $L \times L$ array of pixels. The bad iterative properties of (A) are alleviated through various improvements allowing for high or even infinite contrast. ${ }^{43}$ These schemes are used here. Fast convergence is achieved by taking $\mathbb{L}^{(0)}$ of type (5), with the same anisotropy ratio $k$ as $\mathbb{L}^{(1)}$, but with considerably lower moduli, namely $\kappa^{0} / \kappa \simeq 5.10^{-4}$ and $\mu^{0} / \mu \simeq \lambda^{0} / \lambda$ $\simeq 0.2$ (not necessarily optimal values). The Fourier transform of $G$ reads $^{44}$

$$
G_{i j k l}(\mathbf{q})=-\left\{q_{i}\left[N^{-1}(\mathbf{q})\right]_{j k} q_{l}\right\}_{\mathrm{sym}},
$$

where $\{\cdot\}_{\text {sym }}$ indicates a symmetrization so that $G_{i j k l}=G_{k l i j}$ $=G_{j i k l}$, and where $N_{i j}(q)=q_{k} L_{i k l j}^{(0)} q_{l}$ is the acoustic tensor.

Calculations are carried out for various anisotropy ratios $\left(10^{-4} \leq k \leq 10^{4}\right)$ and porosities $\left(0<f<f_{c}\right)$ using FFT routines. Three sizes $L=512,1024$, and 2048 are considered to monitor size effects. The smallest one leads to results with satisfactory convergence properties, except in cases of high anisotropy, where a better resolution was required to render the fine structure of the field patterns. We used $L=2048$ whenever an appreciable difference was found between $L$ $=512$ and 1024 .

Once the strain $\varepsilon_{i j}(\mathbf{q})$ is computed, the displacement $\mathbf{u}(\mathbf{q})$ is deduced from $(\mathbf{q} \neq 0)$ (Ref. 45)

$$
\begin{gathered}
u_{x}(\mathbf{q})=-i\left\{q_{x}\left[\varepsilon_{x x}(\mathbf{q})-\varepsilon_{y y}(\mathbf{q})\right]+2 q_{y} \varepsilon_{x y}(\mathbf{q})\right\} / q^{2}, \\
u_{y}(\mathbf{q})=i\left\{q_{y}\left[\varepsilon_{x x}(\mathbf{q})-\varepsilon_{y y}(\mathbf{q})\right]-2 q_{x} \varepsilon_{x y}(\mathbf{q})\right\} / q^{2} .
\end{gathered}
$$

Only SS or PS macroscopic strain loadings are considered $\left(\overline{\boldsymbol{\varepsilon}}_{m}=0\right)$. Other shear states follow from linearity. For both modes, the linear elastic problem is solved for various anisotropy ratios $0 \leq k \leq \infty$. Effective moduli are computed using one component at a time, e.g., $\tilde{\lambda}=\left\langle\sigma_{x y}\right\rangle /\left[2\left\langle\varepsilon_{x y}\right\rangle\right]$.

\section{B. Overview: displacement and stress maps}

Typical displacement and stress maps obtained by (isochoric) FFT calculations are as follows. Since first-order infinitesimal displacements are used, it should be borne in mind that however singular, the displacement patterns are at most incipient ones. In limits of infinite anisotropy $k \rightarrow 0, \infty$, the structure of the solutions tends toward one that is schema-

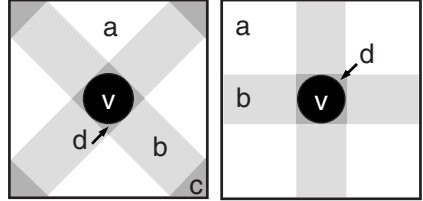

FIG. 2. Structure of unit-cell field patterns for high anisotropy. Left, pattern for SS loading and $k=\infty$. Right, pattern for PS loading and $k=0$. In these figures, $v=$ void; $b, c, d=$ deformation bands in the matrix (not intersecting, intersecting far from the void, and intersecting around the void, respectively); $a=$ remaining parts of the matrix.

tized in Fig. 2, being organized into bands of width one void diameter. Two remarkable types of structures are found, depending on the loading direction and on the type of anisotropy. They differ essentially by the presence of zones in the matrix where the bands cross (denoted by $c$ in Fig. 2).

With the above mentioned caveat, the following approximate symmetry holds between the maps: ${ }^{24}$

$$
\mathcal{R}_{45^{\circ}} \text { (void lattice) } \Leftrightarrow\left\{\begin{aligned}
k & \leftrightarrow 1 / k \\
\text { PS loading } & \leftrightarrow \text { SS loading, }
\end{aligned}\right.
$$

where the $\mathcal{R}$ symbol denotes a $45^{\circ}$ rotation of the lattice of voids, with all other parameters (material constitutive law and loading) conserved. These field structures, already revealed by the analytical calculations of Ref. 24 for infinite anisotropy (to which we refer the reader for further details), are retrieved here for finite, but high, anisotropy.

Table I displays full-field calculations of the reduced (periodic) displacement field $\mathbf{u}^{*}(\mathbf{x}) \equiv \mathbf{u}(\mathbf{x})-\overline{\boldsymbol{\varepsilon}} \cdot \mathbf{x}$, indicated by arrows, superimposed on a representation of the unit cell deformed using a rescaled displacement $\beta \mathbf{u}^{*}(\mathbf{x})$, for anisotropy ratios $k=10^{-3}, 1$, and $10^{3}$ in SS and PS loadings, for a moderate porosity $f=0.1$. To highlight the deformation pattern, the magnification factor $\beta$ lies between 1 and 10. Lighter gray tones in the deformed matrix indicate regions subjected to a strong extension. The unit cell is replicated in order to emphasize the displacement ("flow") pattern. For low and high $k$, the features of the displacement maps are in agreement with the exact results derived at infinite anisotropy in Ref. 24, which they enlighten.

The flow pattern is organized in closed convection cells of square shape, delimited by black boxes. Two types of cells, rotated $\sigma_{\perp}$ with respect to the Cartesian axes, and related by a mirror symmetry, suffice to account for the flow pattern in SS (maps A, B, and C). As a consequence, and due to the high anisotropy, the edges of the unit cells in (A) and (C) undergo nonzero and quasi-piecewise-linear deformation. On the other hand, four types of convection cells, aligned along the Cartesian axes, related by mirror symmetries with respect to these axes, and fully enclosed within one unit cell, are required to produce the flow pattern in PS $(\mathrm{D}, \mathrm{E}, \mathrm{F})$.

Compared to the $k=1$ isotropic solutions of (B) and (E), solutions for highly anisotropic situations are either: (i) localized in strain, with a displacement field discontinuous at places [maps (C) and (D)]; (ii) localized in stress, with con- 
TABLE I. Reduced displacement field $\mathbf{u}^{*}$ (arrows) and resulting elastic deformation of the unit cell (to lowest order of perturbations) in SS and PS loadings for anisotropy ratios $k=10^{-3}, 1$, and $10^{3}$. Four unit cells are represented. An enlargement of a void is shown in map (D).

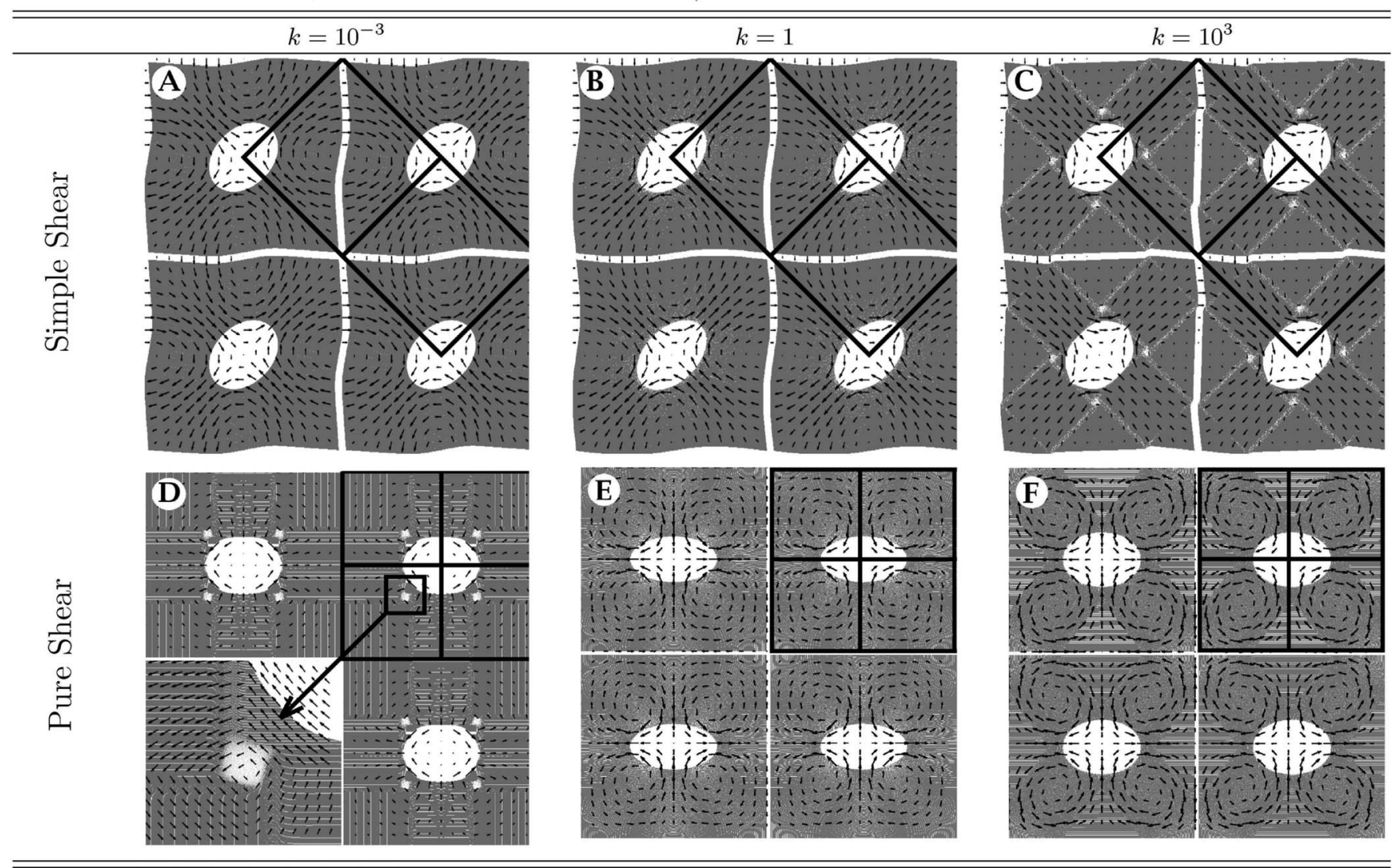

tinuous displacement as in (A) and (F). Strain localization arises whenever loading along a "hard" mode takes place. Then, the highly anisotropic medium resists most the applied strain and undergoes both a high induced stress and a weak induced strain. In the limit of infinite anisotropy, a rigid "block sliding" incipient pattern results, where the flow is organized in bands of width one void diameter (see also Fig. 2 ), where the tangential component of $\mathbf{u}^{*}$ is discontinuous, and where strain concentrates as Dirac distributions along the sliding lines. This pattern is tantamount to a breakdown mechanism. In turn, block sliding leaves four incipient voids in the matrix in (C) and (D), at locations where the sliding lines intersect at $90^{\circ}$. One such void is enlarged in (D). One important difference between cases (C) and (D) is that in (C), the flow bands (of width one void diameter) cross inside the matrix due to their $45^{\circ}$ orientation. Flow redistribution then takes place in the intersection zones. On the contrary, in (D) such zones do not exist in the matrix, and flow redistribution requires a nonzero displacement component normal to the band boundaries. As a result, the gradient of the tangential component of $\mathbf{u}^{*}$ is higher in (C) than in (D).

Solutions with a continuous displacement field are obtained instead when loading is applied along the soft deformation mode. With the remaining deformation mode being harder, this leaves less possibilities for easy deformation than in the isotropic case; this explains why the unit cells of (A) and $(\mathrm{F})$ are much less deformed-a magnification $\beta=10$ is used-than the cells (B) and (E) of the isotropic materialplotted with $\beta=1$.

Table II displays, for $f=0.1$ and for increasing anisotropy ratios $k$, maps of the independent stress components $\sigma_{m}, \sigma_{\|}$, and $\sigma_{\perp}$, under SS and PS loadings. Hereafter, the maps are referred to by their individual number (1 to 30). Each map goes along with its own field scale at its right, in correspondence with the color scale at the extreme right of the rows. The "parallel" $(\|)$ and "perpendicular" $(\perp)$ notations refer to the "direction" of the applied macroscopic loading. The SS and PS shear components of the stress are defined in Eq. (4). In PS loading, we have $\sigma_{\|} \equiv \sigma^{\mathrm{PS}}, \sigma_{\perp} \equiv \sigma^{\mathrm{SS}}$, whereas in SS loading: $\sigma_{\|} \equiv \sigma^{\mathrm{SS}}, \sigma_{\perp} \equiv \sigma^{\mathrm{PS}}$ (hereafter, a similar notation is used for strain components). In both cases, the volume average of the nonparallel components vanishes: $\left\langle\sigma_{\perp}\right\rangle=\left\langle\sigma_{m}\right\rangle=0$. The maps display rescaled stresses, such that $\left\langle\sigma_{\|}\right\rangle=1$. Due to linearity, the strain fields are the same, up to a change of scale (although the scales are different in the parallel and perpendicular directions due to the anisotropy).

The following observations are relevant to the regime of high anisotropy, where the stress patterns follow that of Fig. 2. The zones where bands cross depicted in this figure [either in the matrix (zones $c$ ) or close to the voids (zones $d+v$ )] are places of additive screening or enhancement of the stress. Thus, the parallel stress in zone $c$ of map 20 reaches its highest values there, and is twice that in the two crossing bands [however, a much higher transverse stress is encoun- 
TABLE II. (Color online) Parallel $\left(\sigma_{\|}\right)$, transverse $\left(\sigma_{\perp}\right)$ and mean $\left(\sigma_{m}\right)$ stress field maps for SS and PS loadings, with anisotropy ratios $k=0.01,0.2,1,5$, and 100 (porosity $f=0.1$ ). The stress fields are rescaled such that -3.58 .

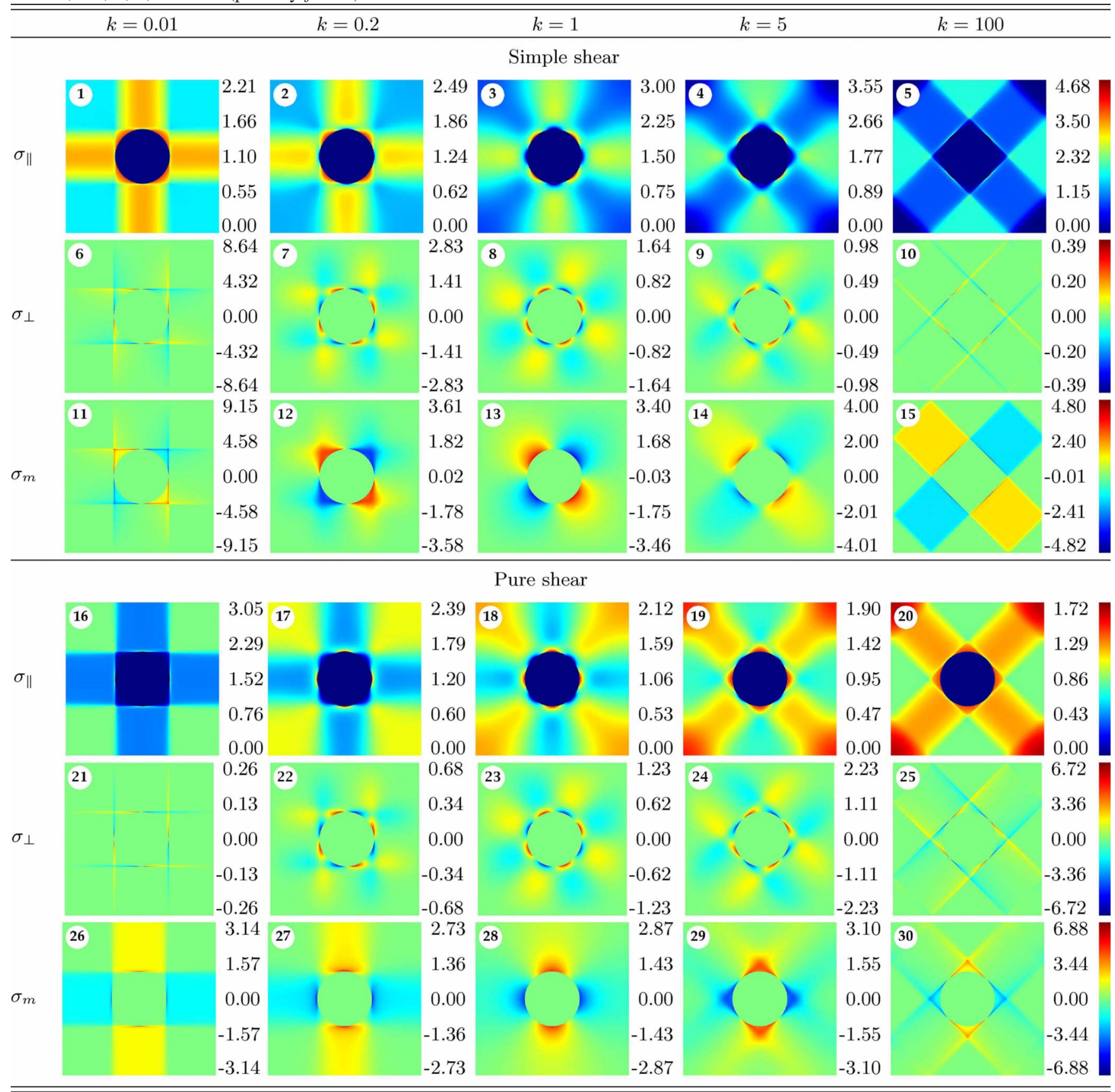

tered in the immediate vicinity of the void (see map 25)]. In a similar way, the vanishing stress in zone $c$ in map 5 is the difference between the stresses in the bands. Two remarks, strictly valid for infinite anisotropy, are in order at this point: First, zones of vanishing stress are squares, of size determined by the void cross section transverse to the bands, so that the disklike shape of the voids is no longer relevant. Second, the buildup of zones of zero stresses (i.e., analogous to porous zones) in the matrix in SS loading leads to an effective doubling of the porosity in the effective shear modulus $\tilde{\lambda}$ at infinite anisotropy, whereby an effective "close packing" threshold, twice as small as the geometric one, ${ }^{25}$ is reached as $f$ increases, leading to a "mechanically advanced" percolative behavior. As a consequence, $\tilde{\lambda}$ decays rapidly with $f$ (see next section).

The stress is less singular than the strain in the limit of infinite anisotropy. Indeed, in a strain-localized situation [loading along a "hard" mode (maps 5, 16)], the displacement is discontinuous. Accordingly, the transverse strain has Dirac singular components along the band frontiers. They abruptly change sign at the special points $( \pm a, 0)$ and $(0, \pm a)$ on the void boundary in PS and at points $( \pm a, \pm a) / \sqrt{2}$ in SS, where $a$ is the void radius. Because of the stress-strain proportionality, these strain singularities can 
be traced in maps 10 and 21 . However, since the perpendicular stress vanishes in the limits $k \rightarrow 0, \infty$, so do its Dirac singularities, as shown by the small values on the scales. The special points, termed hot spots in Ref. 24 are points of extreme matter separation, or crushing, which bear the main cost of the "block sliding" patterns. On the other hand, the incipient secondary voids in maps (C) and (D) of Table I appear (somehow paradoxically) as regions of moderate stress levels.

More generally, the stress field undergoes the following types of singular behavior in the limiting cases of infinite anisotropy:

(i) loading along a hard mode: discontinuous $\sigma_{\|}$component along band frontiers in the direction normal to the frontiers, with finite jump, accompanied by hot spots at the void boundary [maps 5, 16];

(ii) loading along a soft mode: discontinuous derivative of $\sigma_{\|}$in the same direction, with infinite jump [maps 1,20], and discontinuous $\sigma_{\perp}$ with infinite jump across the band frontiers [maps 6, 25].

The mean stress is always singular with the most singular behavior: it has the singularity of the parallel stress in the case of loading along a soft mode, and the singularity of the transverse stress in the case of loading along a hard mode (but the mean strain vanishes in the limit of an incompressible medium).

\section{EFFECTIVE MEDIUM APPROACH}

Nemat-Nasser and Taya ${ }^{26}$ proposed an approximate (dipolar) Fourier-mode approach to the periodic problem, which proved excellent for isotropic components. ${ }^{28,31}$ We apply a similar method to the anisotropic case. More accurate schemes going beyond the dipolar level, however less suitable to analytical treatment, are available. ${ }^{26,29,32}$

The following developments are inspired from a straightforward approach by Suquet, ${ }^{31}$ and result in an EMA of the Clausius-Mossoti, or Maxwell-Garnett (MG) type. ${ }^{27,46-48}$ Its bounding character can be established ${ }^{31}$ using the variational principle of Hashin-Shtrikman ${ }^{49}$ under a form due to Milton and Kohn. ${ }^{50}$ In principle, the technically more involved spectral method of Kantor and Bergman for periodic elastic media ${ }^{27}$ could also have been used to address the present problem. Its actual connection to the present approach and results below however remains to be investigated.

Consider first the general case of a binary composite of volume $V \rightarrow \infty$, the inclusions of which, in proportion to $c^{(2)}$, have an elastic tensor $\mathrm{L}^{(2)}$, and set $\delta L_{L}=\mathrm{L}^{(2)}-\mathrm{L}^{(1)}$. The characteristic function $\chi_{\infty}$ of an infinite periodic array of identical inclusions, of characteristic function $\chi$, is $\chi_{\infty}(\mathbf{x})=\sum_{i} \chi\left(\mathbf{x}-\mathbf{r}_{i}\right)$, where $\mathbf{r}_{i}$ are lattice vectors. Then, $L_{L}=\mathbb{L}^{(1)}+\chi_{\infty} \delta L$. Equations (7) and (8) apply, with $\mathbb{L}^{(0)}=\mathbb{L}^{(1)}$, and $\boldsymbol{\tau}=\chi_{\infty} \delta L_{L}: \boldsymbol{\varepsilon}$. At the dipolar level of approximation, it is assumed that the deformation is a constant in the inclusions so that $\chi_{\infty}(\mathbf{x}) \boldsymbol{\varepsilon}(\mathbf{x})$ is replaced by $\chi_{\infty}(\mathbf{x})\langle\boldsymbol{\varepsilon}\rangle^{(2)}$. This approximation is exact for noninteracting ellipsoidal elastic inclusions only. ${ }^{51}$ Under this approximation, multiplying Eq. (7a) by $\chi_{\infty}(\mathbf{x})$ and integrating over $V$ results in the equation $\langle\boldsymbol{\varepsilon}\rangle^{(2)}=\overline{\boldsymbol{\varepsilon}}-\mathbb{P}: \delta \mathbb{L}_{\mathrm{L}}:\langle\boldsymbol{\varepsilon}\rangle^{(2)}$, or $\langle\boldsymbol{\varepsilon}\rangle^{(2)}=\left(\mathbb{I}+\mathbb{P}: \delta \mathbb{L}^{-1}: \bar{\varepsilon}\right.$, where $\mathbb{P}$ is the Hill depolarization tensor ${ }^{52,53}$ of the inclusion lattice:

$$
\begin{gathered}
\mathbb{P}=-\frac{1}{V c^{(2)}} \int \mathrm{d}^{2} x \mathrm{~d}^{2} x^{\prime} \chi_{\infty}(\mathbf{x}) \mathrm{G}\left(\mathbf{x}-\mathbf{x}^{\prime}\right) \chi_{\infty}\left(\mathbf{x}^{\prime}\right) \\
=-V_{I} \int \frac{\mathrm{d}^{2} q}{(2 \pi)^{2}} \sum_{i} e^{i \mathbf{q} \cdot \mathbf{r}_{i} G(\mathbf{q})\left|\left\langle e^{i \mathbf{x} \cdot \mathbf{q}}\right\rangle_{I}\right|^{2},} \\
=-c^{(2)} \sum_{\substack{\mathbf{q}=2 \pi \mathbf{p} \\
\mathbf{p} \in \text { R.L. }}} G(\mathbf{q})\left|\left\langle e^{i \mathbf{x} \cdot \mathbf{q}}\right\rangle_{I}\right|^{2} .
\end{gathered}
$$

Here, $\langle\cdot\rangle_{I}$ denotes a volume average (with respect to $\mathbf{x}$ ) over one individual inclusion of volume $V_{I}$. The sum in Eq. (10) is over inclusions "centers" in the inclusion lattice (where one inclusion center $\mathbf{r}_{i_{0}}$ coincides with the origin of coordinates). The last equality stems from the Poisson summation formula. The primed sum in Eq. (11) is over nonzero reciprocal lattice vectors $\mathbf{p}=\left(p_{x}, p_{y}\right)$ (with integer components). It should be noted that in the above formulation, the array of inclusions as a whole is considered as one unique (albeit nonconnex) inclusion whose overall depolarization properties are described by $\mathbb{P}$.

In the same way, the average stress $\overline{\boldsymbol{\sigma}}=\langle\mathbb{L}: \boldsymbol{\varepsilon}\rangle$ in $V$ is directly computed from Eqs. (7a) and (7b), by observing that $\int \mathrm{d}^{2} x \mathrm{~d}^{2} x^{\prime} \mathrm{G}\left(\mathbf{x}-\mathbf{x}^{\prime}\right) \chi_{\infty}\left(\mathbf{x}^{\prime}\right): \delta L_{A}: \boldsymbol{\varepsilon}\left(\mathbf{x}^{\prime}\right)=0$ since by definition of $\mathrm{G}, \int \mathrm{d}^{2} x \mathrm{G}(\mathbf{x})=0$. This results in

$$
\overline{\boldsymbol{\sigma}}=\left(\mathbb{L}^{(1)}+c^{(2)} \delta L\right): \overline{\boldsymbol{\varepsilon}}-c^{(2)} \delta L: \mathrm{P}: \delta L:\langle\boldsymbol{\varepsilon}\rangle^{(2)} .
$$

Inserting in this expression the above-obtained $\langle\boldsymbol{\varepsilon}\rangle^{(2)}$, and using definition (6), entails the effective elastic tensor

$$
\widetilde{\mathrm{L}}=\mathbb{L}^{(1)}+c^{(2)} \delta \mathbb{L}_{L}\left(\mathbb{I}+\mathbb{P}: \delta L_{L}\right)^{-1} .
$$

The formula for the void lattice with $\mathbb{L}^{(2)}=0$ follows. For void inclusions, Eq. (13) provides an upper bound to the exact result. $^{31}$

It is convenient to recast Eq. (13) as follows. We separate $\mathbb{P}$ into one- and two-body contributions $\mathbb{P}_{1}$ and $\mathbb{P}_{2},{ }^{54}$ by setting $\mathbb{P} \equiv \mathbb{P}_{1}-c^{(2)} \mathbb{P}_{2}$, where $\mathbb{P}_{1}$ is the $\mathbf{r}_{i_{0}}=0$ term in Eq. (10) and where $\mathbb{P}_{2}$ is defined from the remainder. Introducing $\widetilde{\delta L}=\widetilde{L}-\mathbb{L}^{(1)}$, Eq. (13) takes on, after a simple algebraic transformation, the more familiar form of a MG-type equation for a nonisotropic microstructure: ${ }^{55}$

$$
\widetilde{\mathbb{T}}=c^{(2)} \mathbb{T}_{1},
$$

where

$$
\widetilde{\mathrm{T}} \equiv \tilde{\delta L}:\left(\mathbb{I}+\mathrm{P}_{2}: \delta \tilde{L}^{-1}\right)^{-1}, \quad \mathrm{~T}_{1} \equiv \delta L_{:}:\left(\mathbb{I}+\mathbb{P}_{1}: \delta L_{L}\right)^{-1} .
$$

There, $\mathbb{T}_{1}$ is the elastic polarizability (or $T$ matrix, in the language of multiple scattering theory ${ }^{56,57}$ ) of one single inclusion embedded in the matrix, whereas $\widetilde{T}$ is that of an effective inclusion embedded in the effective medium. The use of different depolarization tensors in $\mathrm{T}_{1}$ and $\mathrm{T}_{2}$ indicates that the effective inclusion has an "elastic shape" determined by two-body correlations and matrix anisotropy, whereas that of the single inclusion solely follows from its own geometrical shape and matrix anisotropy. In classical MG-type approaches, both $\mathbb{P}_{1}$ and $\mathbb{P}_{2}$ (or their dielectric counterparts) are sometimes taken equal ${ }^{47}$ - especially in the prototypical case 


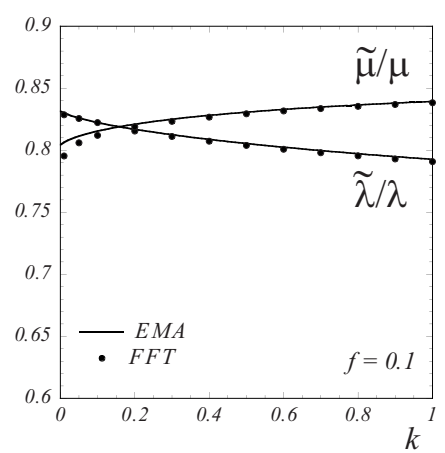

(a)

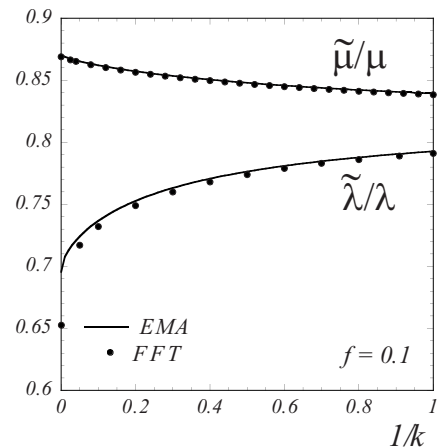

(b)

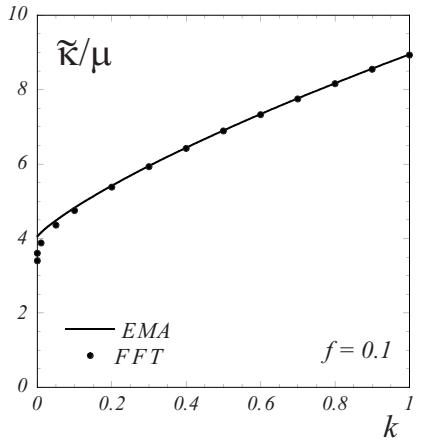

(c)

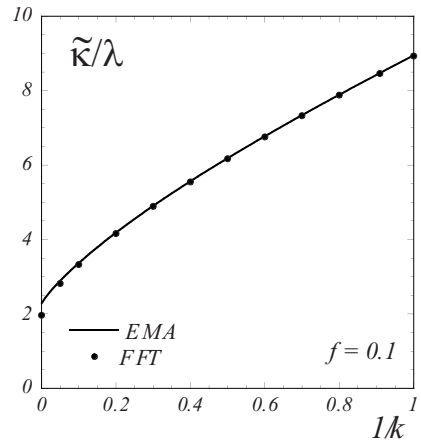

(d)

FIG. 3. Effective shear $(\tilde{\lambda}, \tilde{\mu})$ and compressibility $(\widetilde{\kappa})$ moduli vs anisotropy ratio $k$. Incompressible matrix. Comparisons between the EMA (solid lines) and FFT results (dots) for porosity $f=0.1$. The quantity $\widetilde{\kappa}$ is normalized with respect to the most appropriate modulus, depending on the range of $k$ considered.

of homogeneously distributed spherical inclusions-thus conveying a hypothesis of strong correlations between the shape of the individual inclusions and their spatial organization. In general however, the two depolarization tensors need not be equal (see Refs. 44, 54, and 56 for a systematic discussion of these issues). Note that for ease of interpretation in the MG framework, our definitions of $\mathbb{P}_{1}$ and $\mathbb{P}_{2}$ slightly differ from that of Ref. 54 as to their normalization with respect to the matrix concentration.

The above decomposition of $\mathbb{P}$ proves useful whenever some eigenvalue of $\mathbb{T}_{1}$ should blow up. Then, the corresponding eigenvalue of $\tilde{\delta \mathrm{L}}$, hence of $\widetilde{\mathrm{L}}$, is simply provided by that of $-\mathrm{P}_{2}^{-1}$ as Eqs. (14) and (15) make clear. Further use will be made of $\mathbb{P}_{1,2}$ in Sec. V B.

For cylindrical voids of radius $a$, with $J_{1}$ the Bessel function, $\left\langle e^{i \mathbf{q} \cdot \mathbf{x}}\right\rangle_{I}=2 J_{1}(a q) /(a q)$. Setting

$$
m \equiv \mu / \kappa, \quad \ell \equiv \lambda / \kappa,
$$

one finds from Eqs. (8) and (11) that $P_{i j k l}=\left\{Q_{i p q l} R_{j p q k}\right\}_{\mathrm{sym}}$, where $\mathrm{R}=J-(1+m) \mathbb{E}^{\mathrm{SS}}-(1+\ell) \mathbb{E}^{\mathrm{PS}}$, "sym" denotes a symmetrization with respect to indices $(i, j)$ and $(k, l)$, and

$$
\begin{gathered}
Q=\frac{2}{\pi} \sum_{\mathbf{p} \in \mathrm{R} . \mathrm{L} .}^{\prime} \frac{J_{1}^{2}(2 \pi a p)}{p^{2} \Delta(\mathbf{p})} \mathbf{p} \otimes \mathbf{p} \otimes \mathbf{p} \otimes \mathbf{p}, \\
\Delta(\mathbf{p})=\lambda(1+m)\left(p_{x}^{2}-p_{y}^{2}\right)^{2}+4 \mu(1+\ell) p_{x}^{2} p_{y}^{2} .
\end{gathered}
$$

The reciprocal lattice is a square lattice. Hence $Q$ is also invariant under $D_{4}$. Being completely symmetric, it is of type (1) with $L_{1122}=L_{1212}$ and is determined by two independent scalar lattice sums only. One obtains

$$
\mathrm{Q}=\frac{1}{\mu(1+\ell)}\left[\left(S_{\lambda}+S_{\mu}\right) \mathbb{J}+S_{\mu} \mathbb{E}^{\mathrm{SS}}+S_{\lambda} \mathbb{E}^{\mathrm{PS}}\right],
$$

where after having reduced the lattice sums to sums over the positive quadrant,

$$
\left.\begin{array}{l}
S_{\lambda} \\
S_{\mu}
\end{array}\right\}=\frac{4}{\pi_{\substack{p_{x} \geq 0 \\
p_{y} \geq 1}} \frac{J_{1}^{2}(2 \pi a p)}{p^{2}\left[4 p_{x}^{2} p_{y}^{2}+k\left(p_{x}^{2}-p_{y}^{2}\right)^{2}\right]}}\left\{\begin{array}{c}
\left(p_{x}^{2}-p_{y}^{2}\right)^{2} \\
4 p_{x}^{2} p_{y}^{2}
\end{array} .\right.
$$

These sums bring in the anisotropy parameter

$$
k \equiv[(1+m) \lambda] /[(1+\ell) \mu],
$$

which reduces to $\lambda / \mu$ in the incompressible limit $\kappa \rightarrow \infty$. We remark in passing that

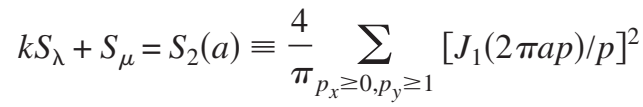

is independent of $k$. After some algebra, one arrives at

$$
\begin{aligned}
\mathbb{P}= & \frac{1}{2 \mu(1+\ell)}\left\{\left(\ell S_{\lambda}+m S_{\mu}\right) J+\left[m S_{\mu}+(1+m) S_{\lambda}\right] \mathbb{E}^{S S}\right. \\
& \left.+\left[\ell S_{\lambda}+(1+\ell) S_{\mu}\right] \mathbb{E}^{\mathrm{PS}}\right\} .
\end{aligned}
$$

The one-body $\mathbb{P}_{1}$ is read from this expression, provided that $S_{\lambda, \mu}$ are computed in the continuum limit, by making the substitutions $\sum \rightarrow \frac{1}{4} \lim _{\varepsilon->0} \int_{\varepsilon}^{\infty} d^{2} q /(2 \pi)^{2}, \mathbf{p} \rightarrow \mathbf{q} /(2 \pi)$ in Eq. (19). Then (in the continuum limit), $S_{\lambda, \mu} \rightarrow$

$$
S_{1 \mu} \equiv \frac{1}{1+\sqrt{k}}, \quad S_{1 \lambda} \equiv \frac{1}{(1+\sqrt{k}) \sqrt{k}} .
$$

Equation (19) shows that $S_{\lambda}$ blows up when $k \rightarrow 0$ due to the contribution of the Cartesian axis $p_{x}=0$. On the other hand, $S_{\mu}$ remains finite or goes to zero in all cases.

The limit of an isotropic matrix where $k=1, \ell=m=\kappa / \mu$ provides $\mathbb{P}_{1}=[2 m J+(1+2 m) \mathbb{K}] /[4 \mu(1+m)]$, where $\mathbb{K} \equiv \mathbb{E}^{S S}$ $+\mathbb{E}^{\mathrm{PS}}$. This expression can be recovered directly from Eq. (8) and from the usual definition in terms of an angular integral ${ }^{58}$ $\mathbb{P}_{1}=-\int d^{2} \Omega_{\hat{q}} G(\hat{\mathbf{q}}) /(2 \pi)$, where $\hat{\mathbf{q}}=\mathbf{q} / q$ (the independence with respect to $\chi$ stems from the rotational symmetry of the voids).

From Eqs. (13) and (22), the effective moduli of the void lattice read, with $f=c^{(2)}$,

$$
\tilde{\kappa} / \kappa=1-f /\left\{1-\left[(\lambda / \mu) S_{\lambda}+S_{\mu}\right] /(1+\ell)\right\},
$$




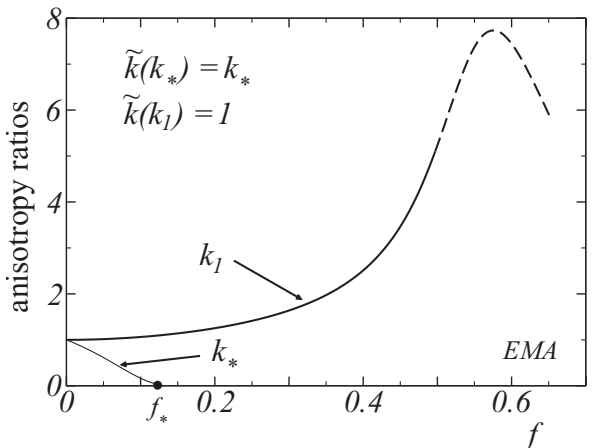

FIG. 4. Anisotropy ratios $k_{1}$ and $k_{*}$ vs porosity $f$ in the EMA. Incompressible matrix.

$$
\begin{gathered}
\tilde{\lambda} / \lambda=1-f /\left\{1-k\left[S_{\lambda}+m S_{\mu} /(1+m)\right]\right\}, \\
\tilde{\mu} / \mu=1-f /\left\{1-\left[S_{\mu}+m S_{\lambda} /(1+\ell)\right]\right\} .
\end{gathered}
$$

Henceforth, incompressibility is assumed for simplicity so that $k \equiv \lambda / \mu$ from now on, unless explicitly stated.

\section{RESULTS}

\section{A. Effective moduli}

The numerical results at various values of $k$ and $f$ discussed in this section are obtained using brute force numerical computations of the sums $S_{\lambda, \mu}$, with convergence checks. The sums $S_{\lambda, \mu}$ are conditionally (and slowly) convergent and the following suitable prescription is used. Sums are carried out over concentric square shells of points $\mathcal{S}_{n}=\left\{\left(p_{x}, n\right) \mid 0\right.$ $\left.\leq p_{x} \leq n-1\right\} \cup\left\{\left(n, p_{y}\right) \mid 1 \leq p_{y} \leq n\right\}$ for $1 \leq n \leq N$, with $N$ sufficiently large. Huge numbers of terms are required for accuracy, especially in the dilute limit.

Figure 3 shows comparisons between the effective moduli computed numerically from the above maps and the effective-medium approximation (EMA) of Sec. IV for $f$ $=0.1$. The agreement is excellent near the case of an isotropic matrix $k=1$ (as is expected for such a small porosity), but also up to high anisotropy. In all cases, the EMA is seen to provide an upper bound for the corresponding full-field esti- mates, as emphasized in the previous section.

Near $k=1$, the orientation of the void lattice makes the medium harder under PS loading than under SS loading [i.e., $\tilde{\mu}(k=1)>\tilde{\lambda}(k=1)]$. Indeed, the anisotropic matrix can be thought of as containing rigidifying fibers (of strength $\mu$ ) oriented at $\sigma_{\|}$along the diagonals that resist PS deformation, and fibers (of strength $\lambda$ ) oriented along the Cartesian axes that resist SS deformation. In the void lattice, the nearestneighboring voids and consequently the largest "directional damage" lie along the Cartesian axes, which explain the difference. We emphasize that while this observation remains true as $k \rightarrow 0$, the situation changes as $k \rightarrow \infty$ : in this limit, due to the presence of the fictitious voids produced by band crossing mentioned in the previous section, the nearestneighboring "voids" become located along the diagonals so that the pure shear (PS) direction becomes, for $k$ higher than some value $k=k_{1}$ (discussed below), the most damaged one, hence the softest.

As $k \rightarrow 0$ [Fig. 3(a)], the curvature of the plots indicates that $\tilde{\mu}$ increases slower than $\mu$, whereas $\tilde{\lambda}$ decreases slower than $\lambda$. As $k \rightarrow \infty$ [Fig. 3(b)], the parts played by $\lambda$ and $\mu$ are reversed. Moreover, the compressibility modulus $\widetilde{\kappa}$ decreases as anisotropy increases in a way comparable to the hardest shear modulus [Figs. 3(c) and 3(d)].

At high anisotropy $k, 1 / k \leqq 0.1$, discrepancies between full-field calculations and the EMA arise for the hardest shear modulus (i.e., $\tilde{\mu}$ when $k \rightarrow 0$, and $\tilde{\lambda}$ when $k \rightarrow \infty$ ), whereas the softest one remains extremely well reproduced. This may indicate that the lattice sums have problems dealing accurately with the effect of second-nearest-neighboring voids. Indeed, the softest direction is always the one where the voids (real or fictitious) are nearest neighbors, whereas the hardest one corresponds to second-nearest neighbors. Note that $\widetilde{\kappa}$, which behaves as the hardest effective modulus, suffers similar discrepancies at high anisotropy.

To discuss the crossing of the curves that takes place in Fig. 3(a), consider the effective anisotropy ratio $\tilde{k} \equiv \tilde{\lambda} / \tilde{\mu}$. Crossing occurs when $\tilde{k}\left(k_{*}\right)=k_{*}$ for some $k=k_{*}(f)$, where the overall medium and the matrix have the same anisotropy ratio. The point $k_{*}$, as estimated by the EMA, is represented vs $f$ in Fig. 4 (no attempt has been made to use full-field

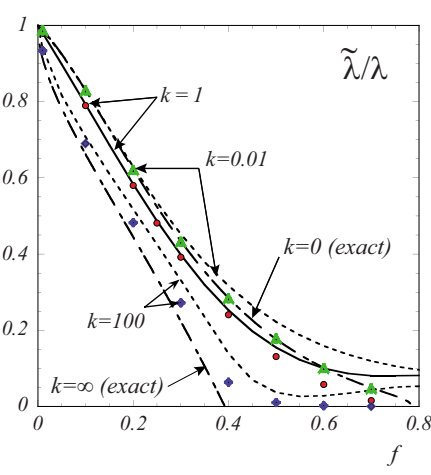

(a)

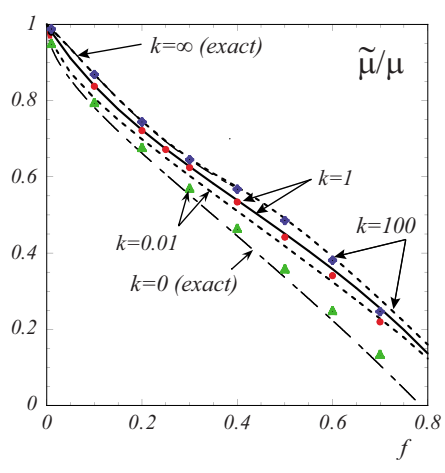

(b)

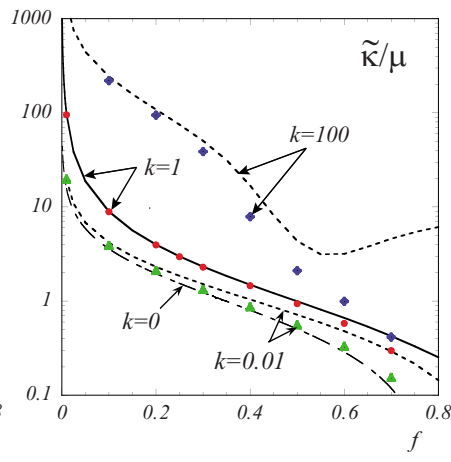

(c)

FIG. 5. (Color online) Effective shear $(\tilde{\lambda}, \tilde{\mu})$ and compressibility $(\widetilde{\kappa})$ moduli vs porosity $f$ for various anisotropy ratio $k$. Incompressible matrix. Comparisons between the EMA for $k=1$ (solid), and $k=0.01,100$ (dotted); FFT results for $k=0.01$ (green triangle dots), $k=0.01$ (red circle dots), and $k=100$ (blue losange dots); and exact results at $k=0, \infty$ taken from Ref. 24 (dash dotted). 
calculations for computational cost reasons). The $k_{*}(f)$ curve shows that crossing only occurs for porosities $f<f_{*} \simeq 0.13$ : as $f$ increases, the curve $\tilde{\lambda} / \lambda$ in Fig. 3(a) goes down to zero faster than $\tilde{\mu} / \mu$, while the crossing point shifts to the left until it vanishes. Remark that $k_{*}<1$ whenever it exists. For $k<k_{*}<1$, the matrix is more anisotropic than the composite; the inverse situation prevails for $k>k_{*}$ and in particular for $f>f_{*}$, where $k_{*} \equiv 0$, so that void-induced anisotropy dominates in this regime.

The other remarkable anisotropy ratio is the aforementioned $k_{1}$, defined by the equation $\widetilde{k}\left(k_{1}\right)=1$, where the overall behavior is isotropic in the plane. This point, also represented on Fig. 4, exists at least up to high porosity values. However, since the EMA is expected to fail around $f=0.5$ (see below), the irrelevant part of the $k_{1}(f)$ curve is sketched with dashed lines in Fig. 4. In the relevant porosity range, the fact that $k_{1}(f)>1$ indicates that the matrix needs to be made harder along the simple shear (SS) $(\lambda)$ mode than along the PS $(\mu)$ mode in order to reach isotropy, so as to compensate for higher softening in this direction due to newly appearing nearest-neighboring voids, as is explained above.

Figure 5 illustrates the behavior of the moduli with the porosity $f$, for finite anisotropy ratios $k=0.01,1$, and 100 , together with the exact results of Ref. 24 at $k=0, \infty$. The exact curve for $\tilde{\mu}$ at $k=\infty$, almost superimposed with the EMA curve for $k=100$ in Fig. 5(b), is available up to $f$ $=\pi / 8$ only. ${ }^{24}$ First, the EMA is again seen to systematically overestimate the moduli. Next, all the elastic moduli must vanish at most at the geometrical close-packing threshold of the voids, ${ }^{25} f=f_{c}=\pi / 4 \simeq 0.78$. The fast Fourier transform (FFT) points in Figs. 5(a) and 5(b) are consistent with this fact, whereas the EMA fails by producing nonzero results at this point. This is not surprising since dipolar EMAs of the Clausius-Mossoti (CM) type are known not to be able to account for percolative-type behavior. ${ }^{25}$ Moreover, the exact result in Fig. 5(a) for $k=\infty$ shows the shear modulus in the hard direction, $\tilde{\lambda}$, to vanish at $f=f_{c} / 2$ due to the fictitious voids produced by band crossing. Accordingly, for large but finite $k, \tilde{\lambda}$ decreases rapidly with $f$ up to $f=f_{c} / 2$, then with a lower slope up to $f=f_{c}$. The EMA again fails to account for the threshold at $f_{c} / 2$, although the local minimum of $\tilde{\lambda}$ at $f$ $\simeq 0.55$ in Fig. 5(a) may indicate that at least part of the phenomenon is captured by the dipolar lattice sums. Interestingly enough, when available, the exact results for infinite anisotropy at $k=0$ (respectively, $k=\infty$ ) are seen to provide tight lower (respectively, upper) bounds to the effective moduli for all values of $k$, and in particular to the isotropic case $k=1$. As far as the effective moduli are concerned, Fig. 5 clearly shows that the EMA can be trusted quantitatively up to $f=0.30$ at most, and is qualitatively reasonable (as long as the matrix is not too anisotropic) up to $f=0.5$.

\section{B. Continuous transition in the dilute limit $f \ll 1$}

\section{Finite anisotropy}

For a finite anisotropy ratio $k$, the dilute expressions for the effective shear moduli at sufficiently small $f$ are read from expressions (24a)-(24c) with $S_{\lambda, \mu}$ replaced by the one- body contributions $S_{1 \lambda}$ and $S_{1 \mu}$ defined in Eq. (23). For the incompressible medium, the EMA estimates of the shear moduli are

$$
\begin{gathered}
\tilde{\lambda} / \lambda=1-f(1+\sqrt{k})+O\left(f^{2}\right), \\
\tilde{\mu} / \mu=1-f(1+1 / \sqrt{k})+O\left(f^{2}\right) .
\end{gathered}
$$

As to the effective compressibility modulus, the incompressible limit leads to the situation described above [Eq. (16)], where one eigenvalue of $\mathbb{T}_{1}$ blows up. This requires us to go beyond the one-body approximation. However, Eq. (A8) in Appendix shows that $S_{2}(a)$ in Eq. (21) is exactly $S_{2}=1-f$ for $f<\pi / 4$. Replacing, e.g., $S_{\mu}$ by $S_{1 \mu}+O(f)$ and computing $S_{\lambda}$ via Eq. (21), then letting $\kappa \rightarrow \infty$ in Eq. (24) [with $k$ read from Eq. (20)] provides

$$
\widetilde{\kappa}=\sqrt{\lambda \mu} / f+O(1) .
$$

Remark that "extended" dilute approximations, which extrapolate the above formulas for moderate anisotropy to finite (but small) porosities, result from taking $\mathrm{P}_{2}=\mathrm{P}_{1}$ in Eq. (15), i.e., from using in Eq. (23),

$$
S_{\mu, \lambda}=S_{\mu, \lambda}^{\mathrm{dil}} \equiv(1-f) S_{1 \mu, \lambda} .
$$

This amounts to assuming pair correlations between the voids dictated by the void shape ${ }^{54}$ and provides expressions of the classical MG type in which the lattice structure is ignored (see Sec. IV).

\section{Infinite anisotropy}

After the incompressible limit $\kappa \rightarrow \infty$ is taken [with $k$ read from Eq. (20)], the limit of infinite anisotropy $k \rightarrow 0$ is obtained by letting $\mu \rightarrow \infty$ in $\tilde{\lambda}$, and $\lambda \rightarrow 0$ in $\tilde{\mu}, \widetilde{\kappa}$. Conversely, $k \rightarrow \infty$ requires $\mu \rightarrow 0$ in $\tilde{\lambda}, \tilde{\kappa}$, and $\lambda \rightarrow \infty$ in $\tilde{\mu}$. In these limits, the sums $S_{\lambda}, S_{\mu}$ in Eq. (19) are computed in Appendix. The obtained effective moduli $\tilde{\lambda}, \tilde{\mu}$, and $\widetilde{\kappa}$ are compared to the exact results of Ref. 24. One finds for $k \rightarrow 0$

$$
\begin{gathered}
\frac{\tilde{\lambda}}{\lambda}=1-f-\frac{32}{3}\left(\frac{f}{\pi}\right)^{3 / 2}+2\left(1-\frac{512}{9 \pi^{3}}\right) f^{2}+O\left(f^{5 / 2}\right), \\
\frac{\tilde{\mu}}{\mu}=1-\frac{3 \pi^{2}}{32}\left(\frac{f}{\pi}\right)^{1 / 2}-\frac{9 \pi^{3}}{1024} f+O\left(f^{3 / 2}\right), \\
\frac{\tilde{\kappa}}{\mu}=\frac{32}{3 \pi^{2}}\left(\frac{\pi}{f}\right)^{1 / 2}-2 \quad \text { (no correction), }
\end{gathered}
$$

while exact expressions are

$$
\frac{\tilde{\lambda}}{\lambda}=1-f-\frac{32}{3}\left(\frac{f}{\pi}\right)^{3 / 2}+\left(1-\frac{6}{\pi}-\frac{8}{\pi^{2}}\right) f^{2}+O\left(f^{5 / 2}\right),
$$

$$
\frac{\tilde{\mu}}{\mu}=1-\left(\frac{f}{\pi}\right)^{1 / 2}-\frac{f}{\pi}+O\left(f^{3 / 2}\right),
$$



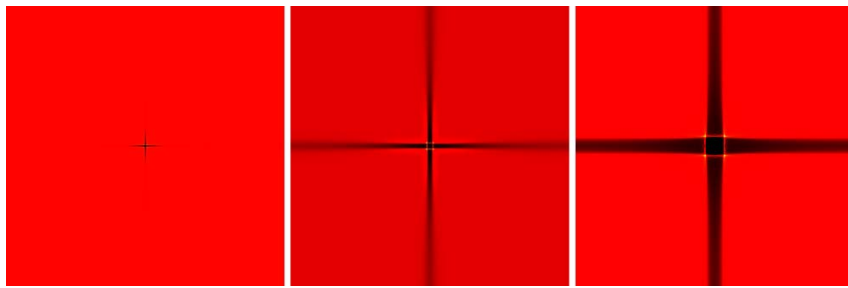

FIG. 6. (Color online) FFT computations of $\varepsilon_{\mathrm{PS}}=\varepsilon_{\|}$in PS loading, for a material with strong anisotropy ratio $k=10^{-3} \ll 1$. Porosities from left to right: $f=f_{r} / 10$ (regular "dilute" regime); $f=f_{r}$ (crossover regime); $f=10 f_{r}$ ("strongly anisotropic" regime), where $f_{r} \simeq k / \pi$ is the crossover porosity. Incompressible matrix. Black represents the highest field values (arbitrary color scale).

$$
\frac{\widetilde{\kappa}}{\mu}=\left(\frac{\pi}{f}\right)^{1 / 2}-2 \text { (no correction). }
$$

For $k \rightarrow \infty$, the EMA estimates read

$$
\begin{gathered}
\frac{\tilde{\lambda}}{\lambda}=1-\frac{3 \pi^{2}}{16 \sqrt{2}}\left(\frac{f}{\pi}\right)^{1 / 2}-\frac{9 \pi^{3}}{512} \frac{f}{\pi}+O\left(f^{3 / 2}\right) \\
\frac{\tilde{\mu}}{\mu}=1-f-\frac{16 \sqrt{2}}{3}\left(\frac{f}{\pi}\right)^{3 / 2}+2\left(1-\frac{256}{9 \pi^{3}}\right)\left(\frac{f}{\pi}\right)^{2}+O\left(f^{5 / 2}\right) \\
\frac{\tilde{\kappa}}{\lambda}=\frac{16 \sqrt{2}}{3 \pi^{2}}\left(\frac{\pi}{f}\right)^{1 / 2}-2 \quad \text { (no correction) }
\end{gathered}
$$

whereas exact expressions are

$$
\begin{gathered}
\frac{\tilde{\lambda}}{\lambda}=1-\left(\frac{2 f}{\pi}\right)^{1 / 2}-\frac{2 f}{\pi}+O\left(f^{3 / 2}\right), \\
\frac{\tilde{\mu}}{\mu}=1-f-\frac{16 \sqrt{2}}{3}\left(\frac{f}{\pi}\right)^{3 / 2}+\left(1-\frac{3}{\pi}-\frac{4}{\pi^{2}}\right) f^{2}+O\left(f^{5 / 2}\right), \\
\frac{\tilde{\kappa}}{\lambda}=\left(\frac{\pi}{2 f}\right)^{1 / 2}-2 \quad \text { (no correction). }
\end{gathered}
$$

The above comparisons show that the EMA estimates do an excellent job of capturing the presence of half-integer powers of $f$ in limits of infinite anisotropy at lowest orders in the dilute limit. Moreover, even when the numerical coefficients are not exact, they are close to the exact values. The less singular character of $\tilde{\lambda}$ in Eqs. (27a) and (27d) when $k \rightarrow 0$ [respectively, $\tilde{\mu}$ in Eqs. (28b) and (28e) when $k \rightarrow \infty$ ] is discussed in Ref. 24.

\section{Dilute transition}

Obviously, a crossover takes place between sets (1) on one hand and (2) [Eq. (27)] on the other hand. Balancing the "extended dilute" sum $S_{\mu}^{\text {dil }}\left[\right.$ Eq. (26)] with $S_{\mu}^{k \rightarrow 0}$ [Eq. (A9)], then with $S_{\mu}^{k \rightarrow \infty}[$ Eq. (A9)] and solving for $k$, provides a discontinuous crossover porosity $f_{r}(k)$ curve, which defines in the $(f, k)$ plane boundary lines between dilute and highanisotropy regions. Owing to the approximations at play, this boundary cannot be trusted for $k$ of order one (for this reason we do not display the curves). On the other hand, we find $f_{r}(k) \simeq\left(9 \pi^{3} / 1024\right) k \simeq k / \pi$ for $k \ll 1$ and $f_{r}(k)$ $\simeq\left(9 \pi^{3} / 512\right) k^{-1} \simeq 2 /(k \pi)$ for $k \gg 1$.

Due to the relation $f=\pi a^{2}$, the crossover porosity in the highly anisotropic regime stems from a length scale -4.82 such that $\xi \sim a / k^{1 / 2}$ for $k \ll 1$, and $\xi \sim a k^{1 / 2}$ for $k \gg 1$. From a mathematical standpoint, these length scales originate from a scaling property of the lattice sums. We focus here on the case $k \rightarrow 0$. The case $k \rightarrow \infty$ can be discussed by adapting this argument. Introducing $K=k /(1-k)$, the sum $S_{\mu}$ in Eq. (19) can be written with a summand proportional to $[1+K \gamma(\hat{\mathbf{p}})]^{-1}$, where the dimensionless quantity $\gamma(\hat{\mathbf{p}})$ reads

$$
\gamma(\hat{\mathbf{p}})=\frac{\left(p_{x}^{2}+p_{y}^{2}\right)^{2}}{4 p_{x}^{2} p_{y}^{2}}
$$

Singling out the contribution of the main diagonal to $S_{\mu}$, the remainder of this sum can be brought down to a sum over $p_{x} \geq 2$ and $1 \leq p_{y} \leq p_{x}-1$, in which $1 / 4 \leq \gamma(\hat{\mathbf{p}}) \leq\left(p_{x} / 1\right)^{2}$. Hence, $\gamma(\hat{\mathbf{p}}) \sim p^{2}$ so that $K \gamma(\hat{\mathbf{p}})$ provides an appreciable $k$-dependent contribution only for $p \gtrsim 1 / \sqrt{K} \sim 1 / \sqrt{k}$. Moreover, $\left[2 J_{1}(x) / x\right]^{2}$ is appreciable only when $x \lesssim 2$. In terms of $p$, this reads $p \lesssim 1 /(\pi a)$ [see Eq. (19)]. Hence $k$-dependent terms contribute provided that $1 / \sqrt{k} \leq p \leq 1 /(\pi a)$. In turn, this is possible only if $\xi(k) \lesssim 1$. For $\xi(k) \gtrsim 1$, a $k$-independent regime instead takes place in $S_{\mu}$.

From a physical standpoint, the length scale $\xi$ represents an effective inclusion size. Figure 6 indeed displays three maps of the parallel strain field in PS loading, computed by FFT at fixed anisotropy ratio $k=10^{-3}$ with varying porosity $f \simeq f_{r} / 10, f_{r}$, and $10 f_{r}$. It is seen that localized shear bands develop from the void as porosity increases. At regime change, they coalesce and span the entire medium. The void can be considered as an isolated inclusion only for $f<f_{r}$. A similar effect takes place for high $k$ values. We checked numerically that in both cases, before coalescence, the strain intensity in the bands (along the band direction) has a strong exponentially decaying component $\varepsilon \propto \exp (-b r / \xi)$, where $r$ is the distance from the void, and $b$ is a numerical coefficient of order one. This component is superimposed on a weak, $f$-dependent, constant, background component that tends to the average value $\bar{\varepsilon}$ as $f \rightarrow 0$.

\section{Average fields and standard deviations}

\section{General considerations}

The first two moments of the fields are required for applications to nonlinear EMAs, and can be consistently computed from any linear homogenization estimate. ${ }^{7}$ Hereafter, $\bar{\varepsilon}_{e}^{(\alpha)} \equiv\langle\varepsilon\rangle^{(\alpha)} / \bar{\varepsilon}$ denotes the phase average of a strain component $\varepsilon$, normalized by the applied macroscopic field. Likewise, we denote by $\operatorname{SD}^{(\alpha)}(\varepsilon)$ its standard deviation (SD) in phase $\alpha$, normalized by $\bar{\varepsilon}$. Similar notations apply to stress components.

The phase-averaged fields in the porous composite are deduced from the set of equations 
TABLE III. SS and PS loadings. Comparisons between EMA estimates (solid lines) and FFT results (dots) at porosity $f=0.1$ for averages of the strain along the loading direction in each phase and standard deviations (SD) of stress and strain components in the matrix vs matrix anisotropy ratio $k=\lambda / \mu$. Strains and stresses are normalized by the appropriate macroscopic component in the loading direction (macroscopic strain $\bar{\varepsilon}=\langle\varepsilon\rangle$ or stress $\bar{\sigma}=\langle\sigma\rangle)$ ). SDs in the voids are irrelevant.

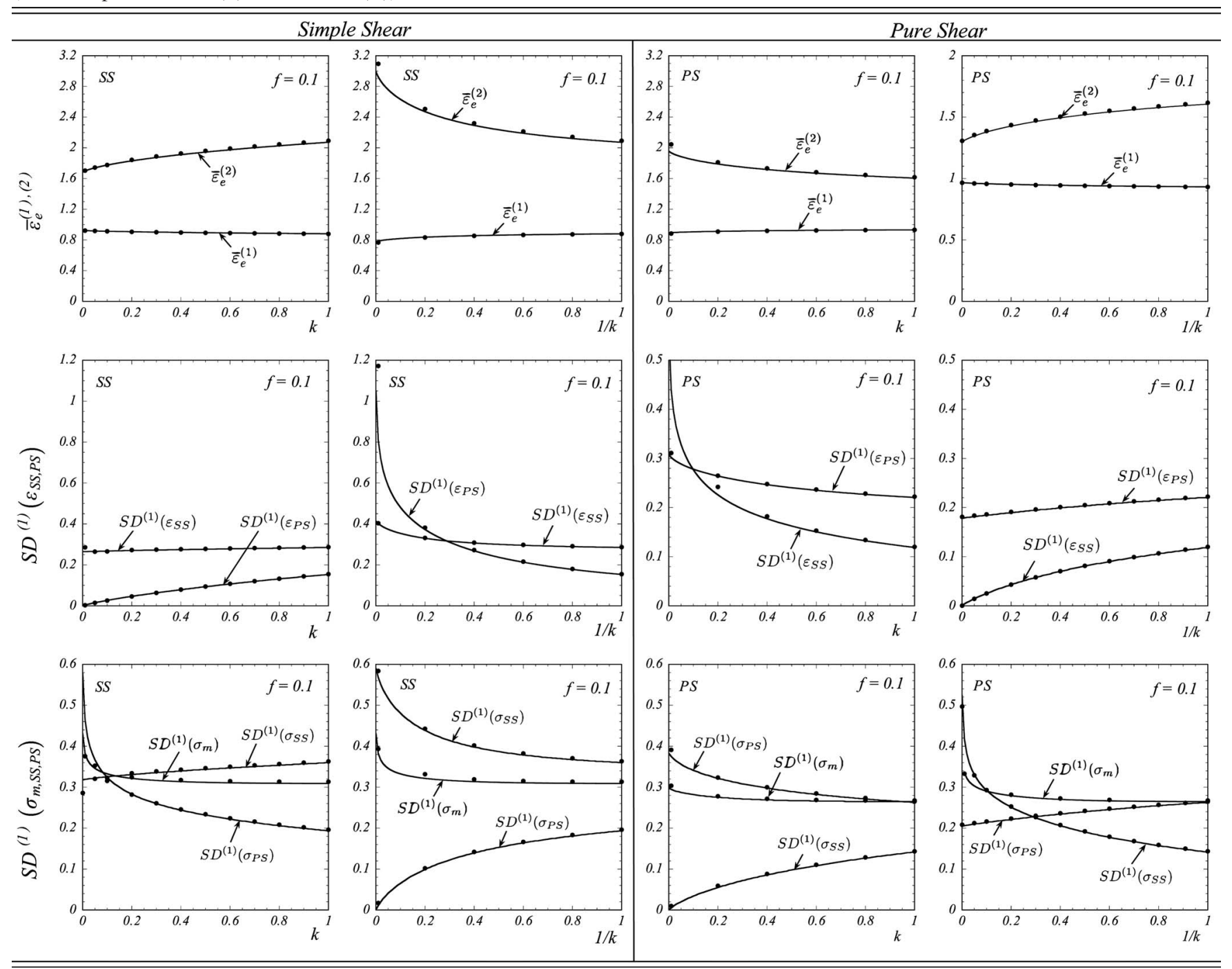

$$
\begin{gathered}
\tilde{\mathbb{L}}: \overline{\boldsymbol{\varepsilon}}=(1-f) \mathbb{L}^{(1)}:\langle\boldsymbol{\varepsilon}\rangle^{(1)}, \\
\overline{\boldsymbol{\varepsilon}}=(1-f)\langle\boldsymbol{\varepsilon}\rangle^{(1)}+f\langle\boldsymbol{\varepsilon}\rangle^{(2)} .
\end{gathered}
$$

Moreover, assuming single mode loading, the second moments in each phase are obtained by taking a derivative of the strain energy with respect to the elastic moduli of the phases, as ${ }^{7,59}$

$$
\left\langle\varepsilon_{\mathrm{m}, \mathrm{SS}, \mathrm{PS}}^{2}\right\rangle^{(\alpha)}=\frac{1}{c^{(\alpha)}} \frac{\partial \tilde{L}}{\partial L^{(\alpha)}}\left\langle\varepsilon_{\mathrm{m}, \mathrm{SS}, \mathrm{PS}}\right\rangle^{2},
$$

where $\tilde{L}$ is $\widetilde{\kappa}$ (respectively, $\tilde{\lambda}$ and $\tilde{\mu}$ ) when the index in the left-hand side is $m$ (respectively, SS and PS) and where $L^{(\alpha)}$ is $\kappa^{(\alpha)}$ (respectively, $\lambda^{(\alpha)}$ and $\mu^{(\alpha)}$ ) when the index in the right-hand side is -4.01 (respectively, SS and PS). The variances follow. If need be, the incompressibility limit is taken after these quantities are computed.
Table III displays for $f=0.1$ the normalized phase-average strains $\bar{\varepsilon}_{e}^{(\alpha)}$ for $\alpha=1,2$ and SDs of the strain and stress components in the matrix, as computed by the EMA and by fullfield calculations. The overall agreement is again excellent, the most important observed deviations, if any, occurring at small $k$. The table layout emphasizes the qualitative correspondence between case $(k, \mathrm{SS})$ and case $(1 / k, \mathrm{PS})$ explained in Ref. 24.

Some trends in the data are explained by appealing to the variational expression of the elastic energy $W$ :

$$
W(\bar{\varepsilon} ; k ; f)=\inf _{\varepsilon \in \mathcal{K}(\bar{\varepsilon})}\left\{\frac{1}{2} \int \varepsilon: \mathrm{L}: \varepsilon\right\}=\frac{1}{2} \bar{\varepsilon}: \widetilde{L}: \bar{\varepsilon},
$$

where $\mathcal{K}(\bar{\varepsilon})=\left\{\varepsilon ; \varepsilon_{i j}=\left(\partial_{i} u_{j}+\partial_{j} u_{i}\right) / 2,\langle\varepsilon\rangle=\bar{\varepsilon}\right\}$ is the set of admissible strain fields. For instance, for an incompressible material under SS loading, Eqs. (30) and (32) imply 
TABLE IV. (Color online) Average shear strains $\bar{\varepsilon}_{e}^{(\alpha)}$ in the matrix $(\alpha=1)$ and in voids $(\alpha=2)$ vs porosity $f$. Comparisons between EMA estimates (solid lines), FFT results (dots), and exact analytical results at $k=0, \infty$ (dash-dotted lines) for particular values of the matrix anisotropy ratio $k=\lambda / \mu$, in pure shear (PS) and simple shear (SS) loadings. The normalization is the same as for Table III (see legend).

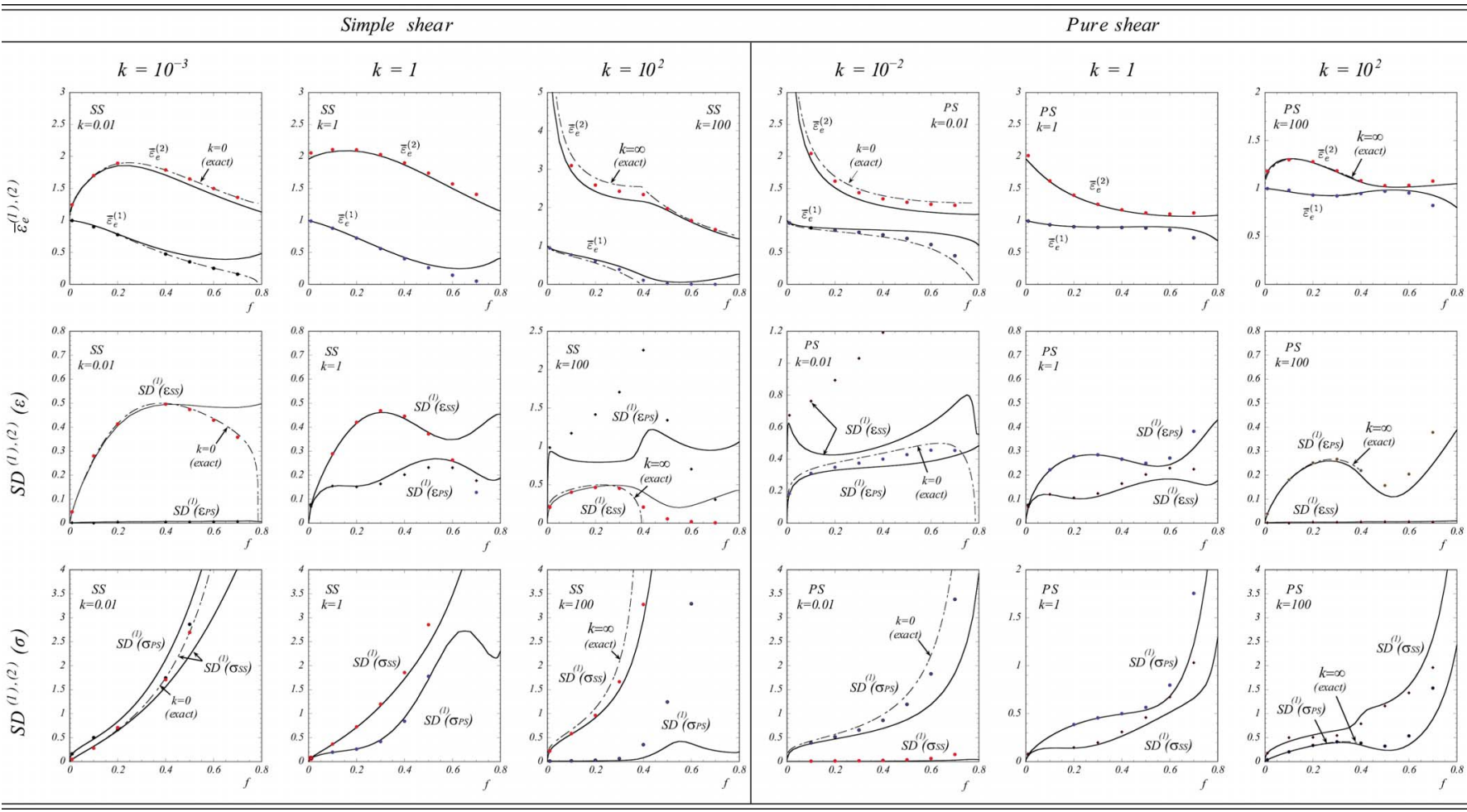

$$
\bar{\varepsilon}\left\langle\varepsilon_{\|}\right\rangle^{(1)}=\left\langle\varepsilon_{\|}^{2}\right\rangle^{(1)}+(1 / k)\left\langle\varepsilon_{\perp}^{2}\right\rangle^{(1)} .
$$

Hence the standard deviation $\mathrm{SD}^{(1)}\left(\varepsilon_{\|}\right)$of the parallel component of the strain is essentially finite since $\left\langle\varepsilon_{\|}\right\rangle^{(1)}$ is, in agreement with the analytical expressions of the SDs in the next section to which we refer the reader for this discussion. Consider now another strain field $\varepsilon^{\prime}$, solution for an anisotropy ratio $k^{\prime}>k$. Using it as a trial field for problem (32) with $k$ provides one inequality. Duplicating the argument with $k$ and $k^{\prime}$, and $\varepsilon$ and $\varepsilon^{\prime}$ interchanged yields after some easy algebra involving Eq. (33),

$$
\mathrm{SD}^{(1)}\left(\varepsilon_{\perp}\right)^{2} \leq \frac{\left\langle\varepsilon_{\|}^{\prime}\right\rangle^{(1)}-\left\langle\varepsilon_{\|}\right\rangle^{(1)}}{\left(1 / k^{\prime}\right)-(1 / k)} \leq \mathrm{SD}^{(1)}\left(\varepsilon_{\perp}^{\prime}\right)^{2},
$$

which entails Eq. (31) for $k^{\prime} \rightarrow k$. Thus, the standard deviation $\mathrm{SD}^{(1)}\left(\varepsilon_{\perp}\right)$ of the transverse (PS) component of the strain field increases with $k$ at $f$ fixed, consistently with Table III. Moreover, using Eq. (34) and the equality $\partial_{k}\left\langle\varepsilon_{\perp}^{2}\right\rangle^{(1)}$ $=-k \partial_{k}\left\langle\varepsilon_{\|}^{2}\right\rangle^{(1)}$ [from Eq. (31)] shows that under SS loading, $\left\langle\varepsilon_{\|}^{2}\right\rangle^{(1)}$ is a decreasing function of $k$. These considerations hold for any fixed microstructure.

Analyzing FFT calculations at $f=0.1$ for various values of $k$ in log-log plots (not shown), we observe that (for this $f$ ) the SDs behave as powers of $k$ with numerical exponents close to $1 / 4$ or $3 / 4$ : For instance, under $\mathrm{SS}$ loading, $\mathrm{SD}^{(1)}\left(\varepsilon_{\perp}\right)$ decays as $k^{3 / 4}$ when $k \rightarrow 0$, and blows up as $k^{1 / 4}$ when $k$ $\rightarrow \infty$; meanwhile, $\mathrm{SD}^{(1)}\left(\sigma_{\perp}\right)=\mathrm{SD}^{(1)}\left(\varepsilon_{\perp}\right) / k \sim k^{-1 / 4}$ as $k \rightarrow 0$ and $\sim k^{-3 / 4}$ as $k \rightarrow \infty$. The "soft" case $k \rightarrow 0$ is in agreement with the dilute analytical expressions [Eqs. (35a) and (35b)] below, which indicates that the computed systems remained in the dilute regime $f \ll f_{r}(k) \sim k$. On the other hand, the "hard" case $k \rightarrow \infty$, where strong strain localization takes place (see map C in Table I), is consistent with Eqs. (35a) and (35b) only if we replace $f$ by $f_{r}(k) \sim k^{-1}$ in these expressions. Thus, here, $\mathrm{SD}^{(1)}\left(\varepsilon_{\perp}\right)$ blows up [see Eq. (36)], but behaves as though the system remained in the crossover regime. This information, extracted numerically, is not contained in expressions (37) and (40) for which we could only produce limiting values.

Actually, in the limit $k \rightarrow \infty$, infinite SDs in the transverse component of the strain result from its concentration as Dirac lines (see Sec. III B), and are linked to discontinuities (jumps) in its parallel component. ${ }^{24}$ This results in a deformation pattern by a "rigid-block-sliding" mechanism, the "rigid blocks" here being connected parts of matter separated by discontinuity lines. This block-sliding effect only takes place provided that the strain jump lines have "percolated." Below percolation, sliding is impossible in a linear material and the transverse strain fluctuations described by Eq. (35) strongly increase with $k$ as $\mathrm{SD}^{(1)}\left(\varepsilon_{\perp}\right) \sim k^{3 / 4}$. On the contrary, beyond percolation, sliding takes place and $\mathrm{SD}^{(1)}\left(\varepsilon_{\perp}\right) \sim k^{1 / 4}$ increases in a weaker way since sliding makes deformation easier. Analogous properties are found under PS loading, provided that $k$ is replaced by $1 / k$ : For instance, $\operatorname{SD}^{(1)}\left(\varepsilon_{\perp}\right)$ is a decreasing function of $k$ and blows up in the hard loading mode as $\sim k^{-1 / 4}$ when $k \rightarrow 0$.

Table IV shows numerical results for the strain and stress field averages and SDs, plotted for various anisotropy ratios $k=0.01,1$, and 100. EMA estimates are provided for com- 
parisons. Except when SDs blow up at strong anisotropy, the EMA estimates are in good agreements with FFT results for porosities up to $f \sim 0.4$. It is worth observing that in situations of high anisotropy ratios, a change in the structure of the strip patterns in the material coincides with a change of concavity of the standard deviations $\operatorname{SD}^{(1)}\left(\varepsilon_{\|}\right)$of the parallel component of the strain field. For instance, when SS loading is applied at $k \gg 1$, the bands cover the whole medium at $f \approx \pi / 8 \approx 0.4$. Around this value, the quantity $\operatorname{SD}^{(1)}\left(\varepsilon_{\|}\right)$ changes from a concave to a convex function of $f$. Such a change also occurs at $f \approx \pi / 8$ for the same SDs when $k \gg 1$ and PS loading is applied. FFT field maps then indicate that the structure of the strain pattern also undergoes an abrupt change at this point (with the appearance of thinner strips linking closest neighboring voids-not shown).

\section{Standard deviations in the dilute limit}

For completeness, we include the standard deviations in the dilute limit (of relevance to nonlinear EMAs) computed from the EMA.

For SS loading, we find for $f \ll f_{r}(k)$ (low anisotropy or low porosity),

$$
\begin{gathered}
\mathrm{SD}\left(\varepsilon_{\|}\right) \sim \operatorname{SD}\left(\sigma_{\|}\right) \sim f^{1 / 2} k^{1 / 4}, \\
\operatorname{SD}\left(\varepsilon_{\perp}\right) \sim f^{1 / 2} k^{3 / 4}, \\
\operatorname{SD}\left(\sigma_{\perp}\right) \sim f^{1 / 2} k^{-1 / 4},
\end{gathered}
$$

where the $f^{1 / 2}$ proportionality of the SDs goes along "classical" $O(f)$ dilute corrections to the effective moduli. In limits of infinite anisotropy, where necessarily $f \gg f_{r}(k)=0$, the EMA estimates provide

$$
\begin{aligned}
& \mathrm{SD}\left(\varepsilon_{\|}\right)=\operatorname{SD}\left(\sigma_{\|}\right) \simeq \frac{4 \sqrt{2}}{\pi^{3 / 4} \sqrt{3}} f^{3 / 4} \\
& +\left(\frac{64 \sqrt{2}}{3 \pi^{9 / 4} \sqrt{3}}-\frac{\sqrt{3} \pi^{3 / 4}}{4 \sqrt{2}}\right) f^{5 / 4}, \quad k \rightarrow 0, \\
& \mathrm{SD}\left(\varepsilon_{\perp}\right)=0, \quad \mathrm{SD}\left(\sigma_{\perp}\right)=\infty, \quad k \rightarrow 0 \\
& \mathrm{SD}\left(\varepsilon_{\|}\right)=\operatorname{SD}\left(\sigma_{\|}\right) \simeq \frac{\pi^{3 / 4} \sqrt{3}}{2^{9 / 4}} f^{1 / 4}, \quad k \rightarrow \infty \\
& \mathrm{SD}\left(\varepsilon_{\perp}\right)=\infty, \quad \operatorname{SD}\left(\sigma_{\perp}\right)=0, \quad k \rightarrow \infty
\end{aligned}
$$

while exact results read $^{24}$

$$
\begin{aligned}
& \operatorname{SD}\left(\varepsilon_{\|}\right)= \operatorname{SD}\left(\sigma_{\|}\right) \simeq \frac{4 \sqrt{2}}{\sqrt{3} \pi^{3 / 4}} f^{3 / 4}+\frac{\sqrt{3} \pi^{3 / 4}}{8 \sqrt{2}} \\
& \times\left(\frac{6}{\pi}+\frac{8}{\pi^{2}}-1\right) f^{5 / 4}, \quad k \rightarrow 0 \\
& \mathrm{SD}\left(\varepsilon_{\perp}\right)=0, \quad \mathrm{SD}\left(\sigma_{\perp}\right)=\infty, \quad k \rightarrow 0 \\
& \mathrm{SD}\left(\varepsilon_{\|}\right)= \mathrm{SD}\left(\sigma_{\|}\right) \simeq(2 f / \pi)^{1 / 4}, \quad k \rightarrow \infty
\end{aligned}
$$

$$
\mathrm{SD}\left(\varepsilon_{\perp}\right)=\infty, \quad \mathrm{SD}\left(\sigma_{\perp}\right)=0, \quad k \rightarrow \infty .
$$

For PS loading, the EMA gives for $f \ll f_{r}(k)$

$$
\begin{gathered}
\mathrm{SD}\left(\varepsilon_{\|}\right) \sim \mathrm{SD}\left(\sigma_{\|}\right) \sim f^{1 / 2} k^{-1 / 4}, \\
\mathrm{SD}\left(\varepsilon_{\perp}\right) \sim f^{1 / 2} k^{-3 / 4}, \\
\mathrm{SD}\left(\sigma_{\perp}\right) \sim f^{1 / 2} k^{1 / 4} .
\end{gathered}
$$

For infinite anisotropy where $f \gg f_{r}(k)=0$, the EMA estimates provide

$$
\begin{gathered}
\mathrm{SD}\left(\varepsilon_{\|}\right)=\mathrm{SD}\left(\sigma_{\|}\right) \simeq \frac{\pi^{3 / 4} \sqrt{3}}{4 \sqrt{2}} f^{1 / 4}, \quad k \rightarrow 0, \\
\mathrm{SD}\left(\varepsilon_{\perp}\right)=\infty, \quad \mathrm{SD}\left(\sigma_{\perp}\right)=0, \quad k \rightarrow 0, \\
\mathrm{SD}\left(\varepsilon_{\|}\right)=\mathrm{SD}\left(\sigma_{\|}\right) \simeq \frac{2^{9 / 4}}{\sqrt{3} \pi^{3 / 4}} f^{3 / 4} \\
+\left(\frac{2^{3 / 4} 32}{3^{3 / 2} \pi^{9 / 4}}-\frac{\sqrt{3} \pi^{3 / 4}}{2^{9 / 4}}\right) f^{5 / 4}, \quad k \rightarrow \infty \\
\mathrm{SD}\left(\varepsilon_{\perp}\right)=0, \quad \mathrm{SD}\left(\sigma_{\perp}\right)=\infty, \quad k \rightarrow \infty,
\end{gathered}
$$

while exact results are ${ }^{24}$

$$
\begin{aligned}
\mathrm{SD}\left(\varepsilon_{\|}\right) & =\mathrm{SD}\left(\sigma_{\|}\right) \simeq(f / \pi)^{1 / 4}, \quad k \rightarrow 0, \\
\mathrm{SD}\left(\varepsilon_{\perp}\right) & =\infty, \quad \mathrm{SD}\left(\sigma_{\perp}\right)=0, \quad k \rightarrow 0, \\
\mathrm{SD}\left(\varepsilon_{\|}\right)= & \mathrm{SD}\left(\sigma_{\|}\right) \simeq \frac{2^{9 / 4}}{\sqrt{3} \pi^{3 / 4}} f^{3 / 4}+\frac{\sqrt{3} \pi^{3 / 4}}{2^{1 / 4} 8} \\
& \times\left(\frac{3}{\pi}+\frac{4}{\pi^{2}}-1\right) f^{5 / 4}, \quad k \rightarrow \infty, \\
\mathrm{SD}\left(\varepsilon_{\perp}\right) & =0, \quad \operatorname{SD}\left(\sigma_{\perp}\right)=\infty, \quad k \rightarrow \infty .
\end{aligned}
$$

Thus, the EMA correctly reproduces the scaling behavior of the SDs in all cases.

\section{CONCLUDING DISCUSSION}

To summarize, we compared the results of an EMA of the Clausius-Mossoti (Maxwell-Garnett) type to FFT calculations, and showed that the agreement is excellent, even in the nontrivial case of localizing behavior as far as effective moduli and averaged fields are concerned, and provided that the void concentration lies below 0.3 . This result is relevant to the study of nonlinear effective-medium techniques: the latter involving both an anisotropic EMA and a specific selfconsistent linearization procedure (which determines the effective anisotropy of the former). The present study shows that strong deviations between FFT and EMA results in nonlinear media should be observed (in the similar setup of a periodic voided medium and in similar conditions of porosity and effective anisotropy). They ought to be attributed to the 
linearization procedure rather than to the underlying liner EMA, even in limits of high effective anisotropy (determined by the field fluctuations in the nonlinear theory). Also, the present work provides a useful independent confirmation of the involved analytical analysis of Ref. 24.

As a by-product of the study, of possible practical applications, we showed that by combining a regular lattice of voids (which makes the structure lighter) and an anisotropic matrix, properties could be tuned so as to make the overall medium elastically isotropic in plane strain.

We also studied analytically the lattice sums which underlie the EMA approach, and showed that they possess a scaling property which, in the dilute limit of small porosity and at high (but finite) anisotropy, allows for a crossover between regular and singular porosity dependence of the effective medium. A length scale $\xi$ was associated to this scaling and interpreted as an effective heterogeneity size. It mathematically diverges in the limit of infinite anisotropy. However, its physically associated counterpart being constrained by the finite size of the cell in the periodic medium, crossover occurs when the effective heterogeneities "percolate," i.e., when $\xi$ is trivially of order one. This corresponds to a strongly correlated regime of strain localization bands spanning the system.

Actually, scaling properties of lattice sums similar to the one considered here, have already been pointed out by Barber, ${ }^{60}$ elaborating on Hall's work, ${ }^{61}$ in a purely mathematical context (in particular, no explanation in terms of length scales was given). Here, we make a connection between this phenomenon and strain localization in anisotropic elastic media. Barber's paper also provides a means to compute the crossover function. However, our lattice sums lead to technical difficulties which preclude the straightforward obtention of a similar result, and we leave this issue for future work.

Moreover, we found that under loading in a "hard" direction of the anisotropic medium, standard deviations of the transverse strain component blow up continuously as a power of the anisotropy ratio, as though the system remained in a crossover regime. This absence of finite threshold for diverging fluctuations and the above-described behavior suggest the existence of a special type of continuous phase transition, of infinite order (called a "weak phase transition" by Hall ${ }^{61}$ ), here obviously without symmetry breaking. The presence of logarithmic terms in $k$ (identified numerically in Appendix) also hints in this direction since logarithmic corrections to scaling constitute a hallmark of transitions of infinite order. ${ }^{62}$ However, a random version of the system should be investigated before reaching definite conclusions.

Finally, it was observed in Ref. 24 that the singular effective moduli in the limit of infinite anisotropy are directly connected to the hyperbolic character of the governing equations in this limit. The very existence of a crossover shows without ambiguity that the problem, although elliptic from a strict mathematical point of view, presents a "quasihyperbolic" character at short distances for high but finite anisotropy. This observation may be of relevance to theoretical investigations of granular materials, for which a model with a similar anisotropic matrix has been proposed. ${ }^{35}$

\section{ACKNOWLEDGMENTS}

The work of M.I.I. and P.P.C. was supported by NSF Grant No. CMS-02-01454. The work of F.W. was supported by a CEA Ph.D. grant. We gratefully thank P. Suquet for having kindly provided to us the notes of Ref. 31 .

\section{APPENDIX: ASYMPTOTICS OF LATTICE SUMS AND DILUTE LIMITS}

We extract the dilute expansions of the lattice sums $S_{\lambda, \mu}$ when $k \rightarrow 0, \infty$ as follows. With the notation

$$
A(a, x)=\left[2 J_{1}(2 \pi a x) / x\right]^{2},
$$

write the lattice sums, with $p=\left(p_{x}^{2}+p_{y}^{2}\right)^{1 / 2}$, as

$$
\begin{gathered}
S_{\lambda}=\frac{1}{\pi} \sum_{\substack{p_{x} \geq 0 \\
p_{y} \geq 1, p_{y} \neq p_{x}}} A(a, p)\left(p_{x}^{2}-p_{y}^{2}\right)^{2} / D(\mathbf{p}), \\
S_{\mu}=\frac{1}{\pi_{p_{x} \geq 1}} \sum_{p_{y} \geq 1} A(a, p) 4 p_{x}^{2} p_{y}^{2} / D(\mathbf{p}),
\end{gathered}
$$

where $D(\mathbf{p})=4 p_{x}^{2} p_{y}^{2}+k\left(p_{x}^{2}-p_{y}^{2}\right)^{2}$. The above expressions explicitly acknowledge the fact that the principal diagonal $p_{x}$ $=p_{y}$ does not contribute to $S_{\lambda}$, and that the Cartesian axis $p_{x}=0$ does not contribute to $S_{\mu}$. Introducing the lattice sums

$$
S_{1}(a)=\frac{1}{\pi} \sum_{n \geq 1} A(a, n), \quad S_{2}(a)=\frac{1}{\pi_{p_{x} \geq 0}} \sum_{p_{y} \geq 1} A(a, p),
$$

provides for $k \rightarrow \infty, 0$

$$
\begin{gathered}
\mathrm{kS}_{\lambda}^{k \rightarrow \infty}=\frac{1}{\pi_{p_{x} \geq 0}} \sum_{p_{y} \geq 1, p_{y} \neq p_{x}} A(a, p)=S_{2}(a)-\frac{1}{2} S_{1}(\sqrt{2} a), \\
S_{\mu}^{k \rightarrow 0}=\frac{1}{\pi_{p_{x} \geq 1}} \sum_{p_{y} \geq 1} A(a, p)=S_{2}(a)-S_{1}(a) .
\end{gathered}
$$

In $S_{\lambda}^{k \rightarrow \infty}$ (respectively, $S_{\mu}^{k \rightarrow 0}$ ) the contribution of the principal diagonal (respectively, Cartesian axis $p_{x}=0$ ) has been subtracted from $S_{2}(a)$. The factors $\sqrt{2}$ and $1 / 2$ result from $n$ being replaced by $\sqrt{2} n$ on the main diagonal.

One privileged tool for exact asymptotic expansions is the Mellin transform. ${ }^{63}$ The transform and its inverse are defined by

$$
\begin{gathered}
M[f(x) ; z]=\int_{0}^{\infty} \mathrm{d} x x^{z-1} f(x), \\
f(x)=\frac{1}{2 i \pi} \int_{c-i \infty}^{c+i \infty} \mathrm{d} z x^{-z} M[f(x) ; z],
\end{gathered}
$$

where $c$ lies within the analyticity strip (parallel to the imaginary axis) of $M[f(x) ; z]$ in the $z$ plane. Shifting the inversion 
contour to the left encircles the poles on the negative $z$ axis and provides the asymptotic series expansion around $x=0$ in positive powers of $x$. Conversely, shifting the contour to the right provides the asymptotic expansion near $x=\infty$ in negative powers of $x$. The Mellin transform provides for $0<c<3$

$$
A(a, p)=\frac{1}{2 i \pi} \int_{c-i \infty}^{c+i \infty} \frac{\mathrm{d} z}{(2 \pi a)^{z-2}} \frac{1}{p^{z}} \frac{2}{\sqrt{\pi}} \frac{\Gamma(z / 2) \Gamma(3 / 2-z / 2)}{\Gamma(2-z / 2) \Gamma(3-z / 2)} .
$$

Next appealing to the definition of the Zeta function for $z$ $>1, \zeta(z)=\sum_{n \geq 1} 1 / n^{z}$, and to Hardy's lattice sum, ${ }^{64,65}$

$$
\sum_{\substack{p_{x} \geq 0 \\ p_{y} \geq 1}} \frac{1}{\left(p_{x}^{2}+p_{y}^{2}\right)^{z / 2}}=\zeta(z / 2) \beta(z / 2), \quad(z>2),
$$

where $\beta(z)=\sum_{n \geq 0}(-1)^{n} /(2 n+1)^{z}$ is the Dirichlet (or Catalan) function, ${ }^{65}$ and interchanging the lattice sums and the contour integral yields

$$
S_{1}(a)=\frac{-i}{\pi^{5 / 2}} \int_{c_{1}-i \infty}^{c_{1}+i \infty} \frac{\mathrm{d} z}{(2 \pi a)^{z-2}} \zeta(z) \frac{\Gamma(z / 2) \Gamma(3 / 2-z / 2)}{\Gamma(2-z / 2) \Gamma(3-z / 2)},
$$

$$
\begin{aligned}
S_{2}(a)= & \frac{-i}{\pi^{5 / 2}} \int_{c_{2}-i \infty}^{c_{2}+i \infty} \frac{\mathrm{d} z}{(2 \pi a)^{z-2}} \zeta(z / 2) \beta(z / 2) \\
& \times \frac{\Gamma(z / 2) \Gamma(3 / 2-z / 2)}{\Gamma(2-z / 2) \Gamma(3-z / 2)},
\end{aligned}
$$

where $1<c_{1}<3$ and where $2<c_{2}<3$ as the result of the above restrictions. The following properties hold: $\Gamma(z)$ has simple poles at negative integers $z=-k \leq 0$ and has no zeros; $\zeta(z)$ has only one simple pole at $z=1$ and has (so-called "trivial") zeros at even, nonzero, negative integers; $\beta(z)$ has no poles and has zeros at odd negative integers. Then, by shifting the contour to the left in both integrals, only the poles $z=1$ and 0 contribute to $S_{1}$, and only the poles at $z$ $=2$ and 0 contribute to $S_{2}$. Eventually we obtain

$$
\begin{gathered}
S_{1}(a)=\frac{32}{3 \pi} a-2 \pi a^{2}, \\
S_{2}(a)=1-\pi a^{2} .
\end{gathered}
$$

The polynomial form of these expressions indicates that they are actually exact since for such functions the asymptotic expansion coincides with the function itself.

The validity of (A8) is linked to the decay of

$$
f(z)=\left|s^{-z} \zeta(z) \frac{\Gamma(z / 2) \Gamma(3 / 2-z / 2)}{\Gamma(2-z / 2) \Gamma(3-z / 2)}\right| \quad \text { as } \mathfrak{R e} z \rightarrow-\infty .
$$

We have in the limit $f(z) \sim s^{-z}|\zeta(z) \Gamma(z / 2) \zeta(z) / \Gamma(-z / 2)|$. Owing to the reflection formula

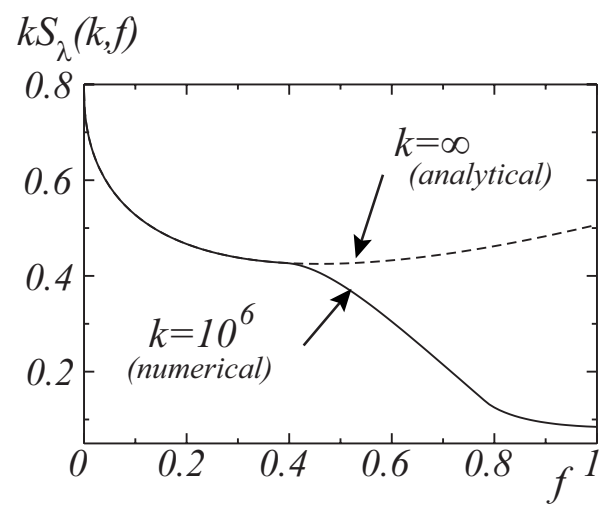

FIG. 7. Quantity $k S_{\lambda}(k, a)$ vs void concentration $f=\pi a^{2}$ as $k \rightarrow \infty$ : comparison between Eq. (A9), dashed line, and a numerical calculation of the lattice sum, solid line, for $k=10^{6}$. Breakdown of Eq. (A9) occurs at $f=\pi / 8 \simeq 0.39$.

$$
\pi^{-z / 2} \Gamma(z / 2) \zeta(z)=\pi^{-(1-z) / 2} \Gamma[(1-z) / 2] \zeta(1-z),
$$

$|\Gamma(z / 2) \zeta(z) / \Gamma(-z / 2)| \sim \pi^{z}|\zeta(-z)| \sim \pi^{z}$. Hence the contribution of the integration line in the limit $c_{1} \rightarrow-\infty$ is negligible only if $s<\pi$. In terms of $a$, this amounts to $a<1 / 2$. The breakdown of the obtained expressions thus corresponds to the close-packing limit $a=1 / 2$. A similar reasoning using the corresponding reflection formula for $\beta(z)$ provides the same range of validity for $S_{2}$.

Combining Eqs. (A5) and (21) then results to

$$
\begin{gathered}
k S_{\lambda}^{k \rightarrow 0}=\frac{32}{3 \pi} a-2 \pi a^{2}, \quad(a<1 / 2) \\
S_{\mu}^{k \rightarrow 0}=1-\frac{32}{3 \pi} a+\pi a^{2}, \quad(a<1 / 2) \\
k S_{\lambda}^{k \rightarrow \infty}=1-\frac{16 \sqrt{2}}{3 \pi} a+\pi a^{2},\left(a<\frac{1}{2 \sqrt{2}}\right)
\end{gathered}
$$

$$
S_{\mu}^{k \rightarrow \infty}=\frac{16 \sqrt{2}}{3 \pi} a-2 \pi a^{2},\left(a<\frac{1}{2 \sqrt{2}}\right) .
$$

The restrictions $a<1 / 2$ and $a<1 /(2 \sqrt{2})$ correspond to critical concentrations $f=\pi / 4$ and $f=\pi / 8$. At these points, either the voids percolate $[k=0$, pure shear (PS) or simple shear (SS) and $k=\infty, \mathrm{SS}]$ or the shear bands undertake a configurational change $(k=\infty, \mathrm{PS}) .^{24}$ An illustration of the breakdown of expression (A9) is provided by Fig. 7.

We could not compute analytically the leading corrections in $k$ to these sums. However, by carefully analyzing brute force numerical computations of the sums for $k$ down to $10^{-5}$ or up to $10^{6}$ for $f=0.1$, we found that corrections to Eqs. (A9a)-(A9d) are of the form $O(k \log k), O(-k \log k)$, $O\left(k^{-1} \log k\right)$, and $O\left(-k^{-1} \log k\right)$, respectively. 
As a final remark, we emphasize that only (divergent) asymptotic series for $S_{1}(a), S_{2}(a)$ at $a>1 / 2$ can be obtained: then, the integrand in both contour integrals blows up as $[z /(4 \pi a e)]^{z}$, where $e$ is Euler's constant. These asymptotic expansions are easily extracted. We do not provide them here since the region $a \gtrsim 1 / 2$ cannot be examined without appealing to additional investigation procedures (e.g., Padé approximants), which lie outside the scope of this paper.
*Present address: Mines Paristech, Centre de Morphologie Mathématique, Mathématiques et Systèmes, 35 rue St-Honoré, F-77305 Fontainebleau Cedex, France; francois.willot@ensmp.fr.

†yves-patrick.pellegrini@cea.fr

${ }^{\ddagger}$ Present address: Departamento de Aeronáutica, Facultad de Ingenería, Universidad Nacional de La Plata, Calle 1 y 47, (1900) La Plata, Argentina; mii23@cam.ac.uk.

§ponte@seas.upenn.edu

${ }^{1}$ X. C. Zeng, D. J. Bergman, P. M. Hui, and D. Stroud, Phys. Rev. B 38, 10970 (1988).

${ }^{2}$ R. Blumenfeld and D. J. Bergman, Phys. Rev. B 40, 1987 (1989).

${ }^{3}$ N. C. Kothari, Phys. Rev. A 41, 4486 (1990).

${ }^{4}$ T. K. Ballabh, M. Paul, T. R. Middya, and A. N. Basu, Phys. Rev. B 45, 2761 (1992).

${ }^{5}$ P. Ponte Castañeda, G. deBotton, and G. Li, Phys. Rev. B 46, 4387 (1992).

${ }^{6}$ P. Ponte Castañeda, J. Mech. Phys. Solids 44, 827 (1996).

${ }^{7}$ P. Ponte Castañeda and P. Suquet, Adv. Appl. Mech. 34, 171 (1998), and references therein.

${ }^{8}$ Y. P. Pellegrini, Phys. Rev. B 64, 134211 (2001).

${ }^{9}$ P. Ponte Castañeda, Phys. Rev. B 64, 214205 (2001).

${ }^{10}$ P. Ponte Castañeda, J. Mech. Phys. Solids 50, 737 (2002); 50, 759 (2002)

${ }^{11}$ R. F. Bishop, R. Hill, and N. F. Mott, Proc. Phys. Soc. London 57, 147 (1945).

${ }^{12}$ K. Weinberg, A. Mota, and M. Ortiz, Comput. Mech. 37, 142 (2006), and references therein.

${ }^{13}$ M. Idiart and P. Ponte Castañeda, C. R. Mec. 333, 147 (2005).

${ }^{14}$ O. Lopez-Pamies and P. Ponte Castañeda, J. Mech. Phys. Solids 54, 807 (2006); 54, 831 (2006).

${ }^{15}$ L. M. Kachanov, Fundamentals of the Theory of Plasticity (Dover, New York, 2004).

${ }^{16}$ S. Roux and D. François, Scr. Metall. Mater. 25, 1087 (1991).

${ }^{17}$ S. Roux and A. Hansen, J. Phys. II France 2, 1007 (1992).

${ }^{18}$ A. Donev, C. E. Musolff, and P. M. Duxbury, J. Phys. A 35, L327 (2002).

${ }^{19}$ J. Boksiner and P. L. Leath, Phys. Rev. E 67, 066610 (2003).

${ }^{20}$ L. Benguigui, Phys. Rev. B 38, 7211 (1988).

${ }^{21}$ D. R. S. Talbot and J. R. Willis, IMA J. Appl. Math. 35, 39 (1985).

${ }^{22}$ P. Ponte Castañeda, J. Mech. Phys. Solids 39, 45 (1991).

${ }^{23}$ P. Suquet, C. R. Acad. Sci., Ser. IIb: Mec., Phys., Chim., Astron. 320, 563 (1995).

${ }^{24}$ F. Willot, Y.-P. Pellegrini, and P. Ponte Castañeda, J. Mech. Phys. Solids 56, 1245 (2008).

${ }^{25}$ S. Torquato, Random Heterogeneous Materials, 2nd ed. (Springer, New York, 2005).

${ }^{26}$ S. Nemat-Nasser and M. Taya, Q. Appl. Math. 39, 43 (1981); 43, 187 (1984) (Erratum).

${ }^{27}$ Y. Kantor and D. J. Bergman, Appl. Phys. Lett. 41, 932 (1982);
J. Mech. Phys. Solids 32, 41 (1984).

${ }^{28}$ K. C. Nunan and J. B. Keller, J. Mech. Phys. Solids 32, 259 (1984).

${ }^{29}$ Ruibao Tao and Ping Sheng, J. Acoust. Soc. Am. 77, 1651 (1985).

${ }^{30}$ A. Sangani and W. Lu, J. Mech. Phys. Solids 35, 1 (1987).

${ }^{31}$ P. Suquet, C. R. Acad. Sci., Ser. II: Mec., Phys., Chim., Sci. Terre Univers 311, 769 (1990); Méthodes de calcul simplifiées pour la détermination des propriétés élastiques de composites à structure périodique (unpublished).

${ }^{32}$ D. J. Bergman and K.-J. Dunn, Phys. Rev. B 45, 13262 (1992).

${ }^{33}$ R. C. McPhedran and A. B. Movchan, J. Mech. Phys. Solids 42, 711 (1994).

${ }^{34}$ V. A. Buryachenko, Int. J. Solids Struct. 42, 4811 (2005).

${ }^{35}$ M. Otto, J.-P. Bouchaud, P. Claudin, and J. E. S. Socolar, Phys. Rev. E 67, 031302 (2003).

${ }^{36}$ D. A. G. Bruggeman, Ann. Phys. 29, 160 (1937).

${ }^{37}$ We emphasize however that for periodic lattices in an isotropic medium noninteger powers of $f$ show up at higher orders, see, e.g., Refs. 25 and 30 and R. C. McPhedran and D. R. McKenzie, Proc. R. Soc. London, Ser. A 359, 45 (1978).

${ }^{38}$ M. Idiart, P. Ponte Castañeda, F. Willot, and Y. P. Pellegrini (unpublished).

${ }^{39}$ G. A. Francfort and P. M. Suquet, Proc. - R. Soc. Edinburgh, Sect. A: Math. 131, 351 (2001)

${ }^{40}$ Specifically, $E=\mu(3-\mu / \kappa), \nu=(\kappa-\mu) /(2 \kappa)$ for plane strain; $E$ $=4 \kappa \mu /(\kappa+\mu), \nu=(\kappa-\mu) /(\kappa+\mu)$ for plane stress; the shear modulus $\lambda$ of the simple shear mode is unchanged.

${ }^{41}$ H. Moulinec and P. Suquet, C. R. Acad. Sci., Ser. IIb: Mec., Phys., Chim., Astron. 318, 1417 (1994).

${ }^{42}$ J. Korringa, J. Math. Phys. 14, 509 (1973).

${ }^{43}$ J. C. Michel, H. Moulinec, and P. Suquet, Comput. Methods Appl. Mech. Eng. 172, 109 (1999); Int. J. Numer. Methods Eng. 52, 139 (2001); H. Moulinec and P. Suquet, Physica B (Amsterdam) 338, 58 (2003).

${ }^{44}$ J. R. Willis, J. Mech. Phys. Solids 25, 185 (1977).

${ }^{45} \mathrm{~F}$. Willot and Y. P. Pellegrini, in Continuum Models and Discrete Systems CMDS 11, edited by D. Jeulin and S. Forest (École des Mines, Paris, 2008), p. 443.

${ }^{46}$ J. C. Maxwell Garnett, Philos. Trans. R. Soc. London, Ser. A 203, 385 (1904).

${ }^{47}$ R. W. Cohen, G. D. Cody, M. D. Coutts, and B. Abeles, Phys. Rev. B 8, 3689 (1973).

${ }^{48}$ The form is also that of the "Quasi-Crystalline Approximation," exact here as far as the correlations are concerned. See, e.g., U. Frisch, in Probabilisitc Methods in Applied Mathematics, edited by A. T. Bharucha-Reid (Academic, New York, 1968), Vol. 1, p. 75.

${ }^{49}$ Z. Hashin and S. Shtrikman, J. Mech. Phys. Solids 11, 127 (1963).

${ }^{50}$ G. Milton and R. V. Kohn, J. Mech. Phys. Solids 36, 597 (1988). 
${ }^{51}$ J. D. Eshelby, Proc. R. Soc. London, Ser. A 241, 376 (1957).

${ }^{52}$ R. Hill, J. Mech. Phys. Solids 13, 89 (1965).

${ }^{53}$ J. R. Willis, Adv. Appl. Mech. 21, 1 (1981).

${ }^{54}$ P. Ponte Castañeda and J. R. Willis, J. Mech. Phys. Solids 43, 1919 (1995).

${ }^{55}$ G. A. Niklasson and C. G. Granquist, J. Appl. Phys. 55, 9 (1980), and references therein.

${ }^{56}$ J. E. Gubernatis and J. A. Krumhansl, J. Appl. Phys. 46, 1875 (1975).

${ }^{57}$ T. R. Middya, M. Paul, and A. N. Basu, J. Appl. Phys. 58, 4095
(1985).

${ }^{58}$ G. Kneer, Phys. Status Solidi 9, 825 (1965).

${ }^{59}$ D. J. Bergman, Phys. Rep., Phys. Lett. 43, 377 (1978).

${ }^{60}$ M. N. Barber, J. Phys. A 10, 2133 (1977).

${ }^{61}$ G. L. Hall, J. Stat. Phys. 14, 521 (1976).

${ }^{62}$ M. Weigel and W. Janke, J. Phys. A 38, 7067 (2005).

${ }^{63} \mathrm{R}$. Wong, Asymptotic Approximations of Integrals (Academic, Boston, 1989).

${ }^{64}$ G. H. Hardy, Mess. Math. 49, 85 (1919).

${ }^{65}$ M. L. Glasser, J. Math. Phys. 14, 409 (1973). 